\title{
Polybrominated Diphenyl Ethers and Gut Microbiome Modulate Metabolic Syndrome-Related Aqueous Metabolites in Mice ${ }^{\sqrt{\$}}$
}

\author{
David K. Scoville, Cindy Yanfei Li, Dongfang Wang, Joseph L. Dempsey, Daniel Raftery, \\ Sridhar Mani, Haiwei Gu, ${ }^{\star}$ and Julia Yue Cui*
}

Department of Environmental and Occupational Health Sciences (D.K.S., C.Y.L., J.L.D., J.Y.C.) and Northwest Metabolomics Research Center, Department of Anesthesiology and Pain Medicine (D.W., D.R.), University of Washington, Seattle, Washington; Department of Laboratorial Science and Technology, School of Public Health, Peking University, Beijing, P. R. China (D.W.); Albert

Einstein College of Medicine, Bronx, New York (S.M.); and Arizona Metabolomics Laboratory, School of Nutrition and Health

Promotion, College of Health Solutions, Arizona State University, Phoenix, Arizona (H.G.) Primary laboratories of origin.

Received January 23, 2019; accepted May 7, 2019

\begin{abstract}
Polybrominated diphenyl ethers (PBDEs) are persistent environmental toxicants associated with increased risk for metabolic syndrome. Intermediary metabolism is influenced by the intestinal microbiome. To test the hypothesis that PBDEs reduce hostbeneficial intermediary metabolites in an intestinal microbiomedependent manner, 9-week old male conventional (CV) and germfree (GF) C57BL/6 mice were orally gavaged once daily with vehicle, BDE-47, or BDE-99 (100 $\mu \mathrm{mol} / \mathrm{kg})$ for 4 days. Intestinal microbiome (16S rDNA sequencing), liver transcriptome (RNA-Seq), and intermediary metabolites in serum, liver, as well as small and large intestinal contents (SIC and LIC; LC-MS) were examined. Changes in intermediary metabolite abundances in serum, liver, and SIC, were observed under basal conditions (CV vs. GF mice) and by PBDE exposure. PBDEs altered the largest number of metabolites in the LIC; most were regulated by PBDEs in GF conditions. Importantly,
\end{abstract}

intestinal microbiome was necessary for PBDE-mediated decreases in branched-chain and aromatic amino acid metabolites, including 3-indolepropionic acid, a tryptophan metabolite recently shown to be protective against inflammation and diabetes. Gene-metabolite networks revealed a positive association between the hepatic glycan synthesis gene $\alpha$-1,6-mannosyltransferase (Alg12) mRNA and mannose, which are important for protein glycosylation. Glycome changes have been observed in patients with metabolic syndrome. In LIC of CV mice, 23 bacterial taxa were regulated by PBDEs. Correlations of certain taxa with distinct serum metabolites further highlight a modulatory role of the microbiome in mediating PBDE effects. In summary, PBDEs impact intermediary metabolism in an intestinal microbiome-dependent manner, suggesting that dysbiosis may contribute to PBDE-mediated toxicities that include metabolic syndrome.

\section{Introduction}

Polybrominated diphenyl ethers (PBDEs) have been added to a myriad of consumer products as flame retardants (2001). Although there are 209 predicted PBDE congeners stemming from the degree of bromination and stereochemistry, three commercial formulations were manufactured and used in the United States (2017). One mixture, known as commercial pentaBDE and sold under several trade names, was primarily applied to furniture cushion foam and carpet pads. OctaBDE and decaBDE mixtures, also sold under different trade names, were primarily applied to plastic components of computers, televisions, and other electronics (2017).

This work was supported by the National Institutes of Health [Grants R01 GM111381, R01 ES030197, R01 ES025708], the University of Washington Center for Exposures, Diseases, Genomics, and Environment [P30 ES0007033], and the Sheldon Murphy Endowment.

https://doi.org/10.1124/dmd.119.086538.

S This article has supplemental material available at dmd.aspetjournals.org.
Despite the fact that commercial formulations are no longer produced in, or imported into, the United States, PBDEs are ubiquitous in human serum and breast milk samples. Recent data from the Methods Advancement for Milk Analysis (MAMA) Study showed that the median serum concentration of BDE-47 was $18.6 \mathrm{ng} / \mathrm{g}$ lipid and $3.9 \mathrm{ng} / \mathrm{g}$ lipid for BDE-99 (Marchitti et al., 2017). Median breast milk concentrations for BDE-47 were observed in the same study to be $31.5 \mathrm{ng} / \mathrm{g}$ lipid, and $6 \mathrm{ng} / \mathrm{g}$ lipid for BDE-99. One route of exposure is from household and workplace dust that is contaminated from PBDEcontaining products ((Hites, 2004, US-EPA, 2012, 2017)). In addition, PBDEs are environmentally persistent and bioaccumulative, facilitating human exposures through diet, especially dairy, fish, and meat ((Schecter et al., 2004, 2010, 2017)). Two commercial PBDE formulations (e.g., DE-71 and Bromkal 70-5DE) that were analyzed for individual congeners consisted of $36 \%-42 \%$ BDE-47 (tetrabrominated), as well as $42 \%-48 \%$ BDE-99 (pentabrominated); thus, BDE-47 and BDE-99 account for approx. 80\%-90\% of total PBDEs in these mixtures (Sanders et al., 2005; LaA Guardia et al., 2006). BDE-47 and BDE-99 are also two of the most prevalent PBDE congeners found in human

ABBREVIATIONS: ACN, acetonitrile; Alg12, $\alpha$-1,6-mannosyltransferase; BCAA, branched-chain amino acid; BSCFA, branched short-chain fatty acid; CV, conventional; FDR, false discovery rate; Gck, glucokinase; Gclc, glutamate-cysteine ligase catalytic subunit; GEO, Gene Expression Omnibus; GF, germ-free; Gnmt, glycine N-methyltransferase; 3-IPA, 3-indoleproprionic acid; LIC, large intestinal contents; Nqo1, NAD(P)H dehydrogenase, quinone 1; PBDE, polybrominated diphenyl ether; RT-qPCR, quantitative reverse transcription-polymerase chain reaction; SIC, small intestinal contents. 


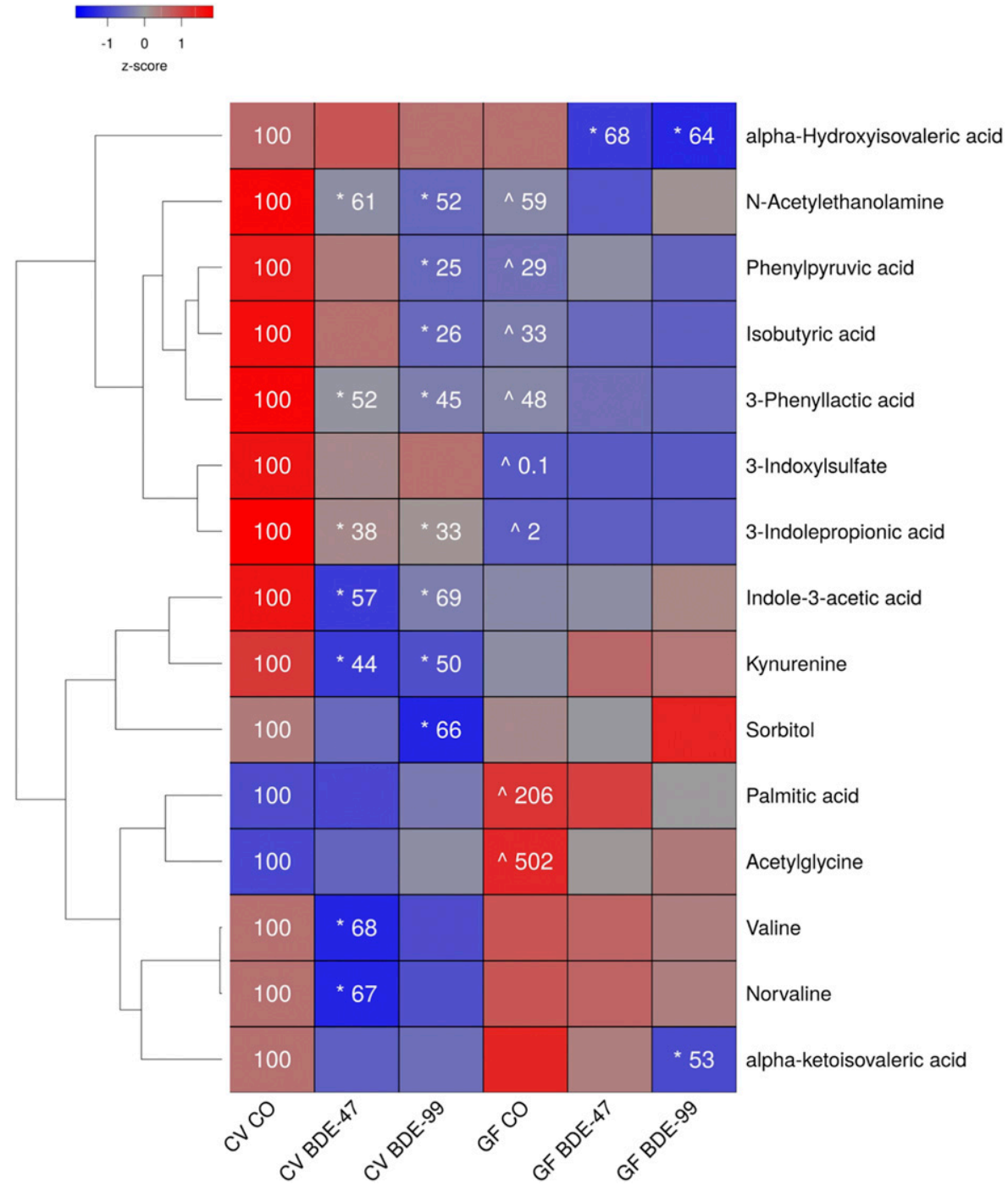

Fig. 1. Two-way hierarchical clustering dendrogram of aqueous metabolites measured using LC-MS that were differentially regulated across treatment groups in serum of $\mathrm{GF}$ and $\mathrm{CV}$ mice following exposures to BDE-47 and BDE-99. Euclidian distance and complete linkage were used to generate the dendrogram. Red indicates higher standardized mean metabolite relative abundances ( $z$-scores) and blue lower. Asterisks $(*)$ represent statistically significant differences between corn oil-treated and PBDE treated groups within enterotype (adjusted $P$ value $<0.05$, Tukey's HSD post-hoc test). Caret signs $(\wedge)$ represent statistically significant baseline differences between $\mathrm{CV}$ and $\mathrm{GF}$ mice. Percent change from CVCO are shown for treatment groups with significant changes.

samples (Sjödin et al., 2001; Schecter et al., 2003; Imm et al., 2009; Marchitti et al., 2017).

Furthermore, recent epidemiologic and animal studies have associations between PBDEs and signs of diabetes and metabolic syndrome. In two independent case-control studies, serum concentrations of BDE-47 in participants with diabetes were significantly associated with increased risk of diabetes (Zhang et al., 2016). In a study among obese individuals, adipose tissue concentrations of both BDE-47 and BDE-99 were significantly elevated in insulin-resistant participants compared with insulin-sensitive participants (Helaleh et al., 2018). BDE-47 and BDE99 concentrations were also associated with adipose tissue concentrations of insulin and interleukin-6. BDE-47 was associated with alkaline phosphatase, which is a biomarker for dysfunction in hepatobiliary secretion, and has also been shown to interfere with rodent glucose homeostasis (McIntyre et al., 2015; Zhang et al., 2016). Rats exposed to DE-71, one of the commercial PBDE mixtures made up primarily of BDE-47 and BDE-99, as well as other minor constituents exhibited changes in liver gene expression patterns that matched patterns associated with metabolic syndrome (Sanders et al., 2005; Dunnick et al., 2012). However, research into mechanisms for PBDE-mediated changes in intermediary metabolism is ongoing.

The dynamic and diverse populations of microbes that populate the lower gastrointestinal tract, collectively known as the intestinal or gut microbiome, are known to interact with host intermediary and xenobiotic metabolism (Nicholson et al., 2012; Li et al., 2017). We have previously shown that the intestinal microbiome modifies PBDEmediated effects on bile acids as well as the expression of genes involved in xenobiotic biotransformation ( $\mathrm{Li}$ et al., 2017, 2018). However, little is known regarding PBDE-mediated intermediary metabolic changes in rodents, and a potential modulatory role for PBDE in the intestinal microbiome. Thus, in this study we integrated transcriptomics, targeted metabolomics, and microbial $16 \mathrm{~S}$ rDNA profiling to evaluate the effects of PBDEs on intermediary metabolism, and we tested our hypothesis that PBDEs downregulate beneficial intermediary metabolites in an intestinal microbiome-dependent manner.

\section{Materials and Methods}

Chemicals. BDE-47 (cat. no. N-105220-10mg, CAS no. 5346-43-1) and BDE-99 (cat. no. FF-BDE-099N-80mg, CAS no. 60348-60-9) were purchased from Chem Service, Inc. (West Chester, PA), and Accustandard, Inc. (New Haven, CT), respectively. Phosphate-buffered saline (pH 7.4, cat. no. 10010023) was purchased from Thermo Fisher Scientific (Waltham, MA). Deionized (DI) water was provided in-house by a Synergy Ultrapure Water System from EMD Millipore (Billerica, MA). The unlabeled metabolite standards were purchased from Sigma-Aldrich (St. Louis, MO) and Fisher Scientific (Pittsburgh, PA) and labeled standards were purchased from Cambridge Isotopes (Tewksbury, MA) 


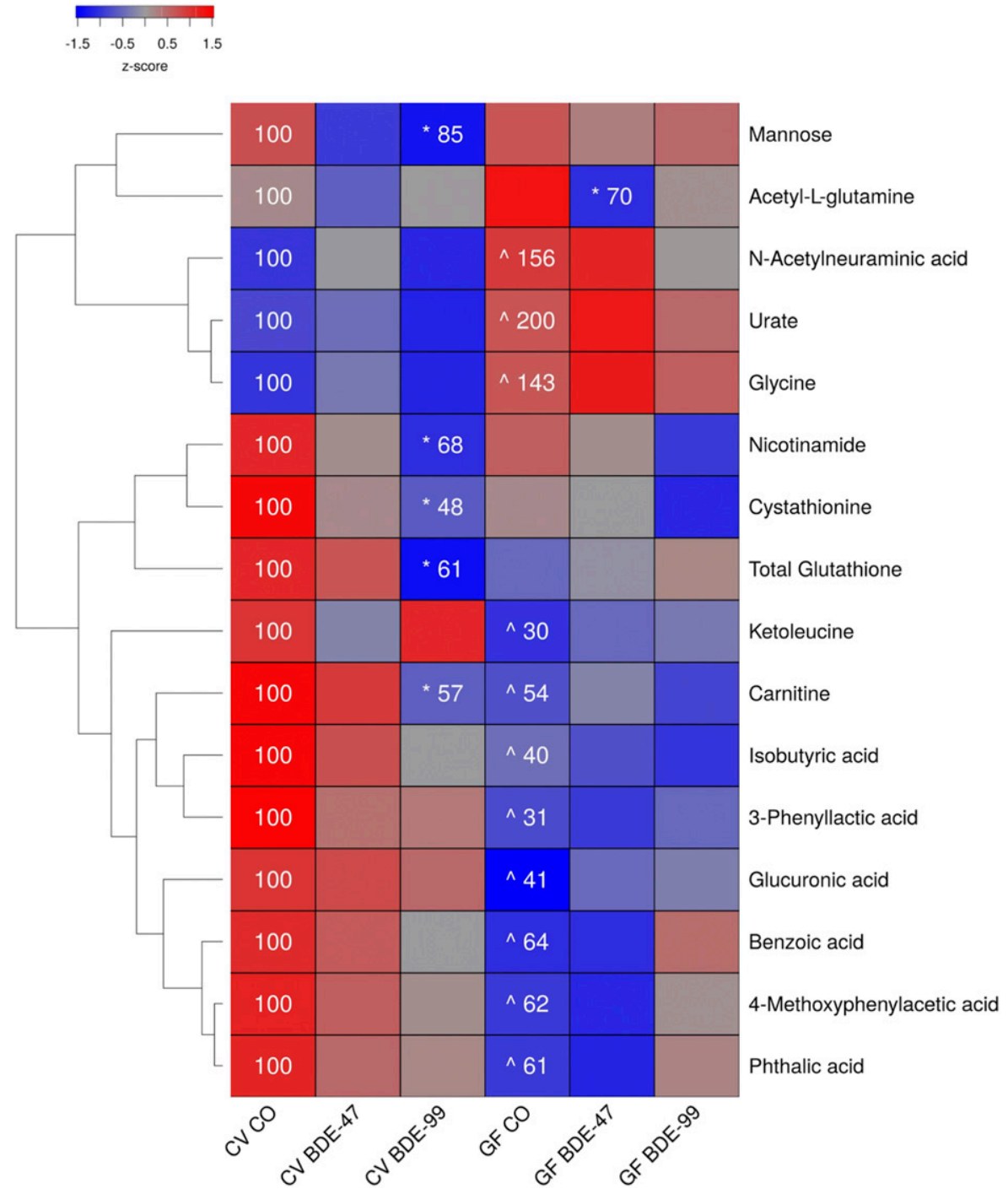

Fig. 2. Two-way hierarchical clustering dendrogram of aqueous metabolites measured using LC-MS that were differentially regulated across treatment groups in liver of GF and $\mathrm{CV}$ mice following exposures to BDE-47 and BDE-99. Euclidian distance and complete linkage were used to generate the dendrogram. Red indicates higher standardized treatment group mean metabolite relative abundances ( $z$-scores) and blue lower. Asterisks (*) represent statistically significant differences between corn oil-treated and PBDE treated groups within enterotype (adjusted $P$ value $<0.05$, Tukey's HSD post-hoc test). Caret signs $(\wedge)$ represent statistically significant baseline differences between $\mathrm{CV}$ and $\mathrm{GF}$ mice. Percent change from $\mathrm{CVCO}$ are shown for treatment groups with significant changes.

(Zhu et al., 2014). Ammonium acetate [cat. no. A11450, CAS no. 631-61-8; liquid chromatography-mass spectrometry (LC-MS) grade] and acetic acid (cat. no. A11350, CAS no. 64-19-7; LC-MS grade) came from Fisher Scientific. Acetonitrile (ACN; cat. no. 1.00029, CAS no. 75-05-8) and methanol (cat. no. 1.06035, CAS no. 67-56-1) both LC-MS grade, ammonium bicarbonate (cat. no. 09830, CAS no. 1066-33-7; purity $>99.99 \%$ ), and all other chemicals and reagents, unless indicated otherwise, were purchased from Sigma Aldrich.

Animals. As previously reported ( $\mathrm{Li}$ et al., 2017, 2018) and briefly summarized here, 8-week-old male C57BL/6J CV mice were purchased from the Jackson Laboratory (Bar Harbor, ME). Mice were acclimated at the University of Washington for 1 week prior to experiments. The initial germ-free (GF) mice, which are on a C57BL/6J background, were purchased from the National Gnotobiotic Rodent Resource Center (University of North Carolina, Chapel Hill, NC). Breeding and colony management of GF mice was performed by the University of Washington Gnotobiotic Animal Core. All mice were housed according to the Association for Assessment and Accreditation of Laboratory Animal Care International guidelines. At 9 weeks of age, the mice $(n=5$ per group) were exposed to vehicle (corn oil, $10 \mathrm{ml} / \mathrm{kg}$ ), BDE-47 [48.5 mg/kg $(100 \mu \mathrm{mol} / \mathrm{kg}$ )], or BDE-99 [56.5 mg/kg (or $100 \mu \mathrm{mol} / \mathrm{kg}$ )] via oral gavage once daily for 4 consecutive days. All mice were euthanized using $\mathrm{CO}_{2}$ narcosis followed by cardiac puncture 24 hours after the final PBDE dose. During the cardiac puncture, blood was collected from the heart with a 25-gauge needle and put into a serum separator tube. Serum was collected and stored at $-80^{\circ} \mathrm{C}$ after centrifugation for 10 minutes at $2000 \mathrm{~g}$ at $4^{\circ} \mathrm{C}$. Liver tissue was frozen in liquid nitrogen and stored at $-80^{\circ} \mathrm{C}$ for further analysis. Small (SIC) and large (LIC) intestinal contents were collected as a pellet first by flushing the lumen with $10 \mathrm{mM}$ dithiothreitol in PBS (Sigma Aldrich) and then the luminal contents were centrifuged at 20,000 $\mathrm{g}$ for 30 minutes at $4^{\circ} \mathrm{C}$. All animal studies were approved by the Institutional Animal Care and Use Committee at the University of Washington.

RNA Isolation and Sequencing. RNA isolation and sequencing methods were previously reported ( $\mathrm{Li}$ et al., 2017). Briefly, total RNA was isolated from frozen liver tissue ( $n=2-4$ /group) using RNA-Bee (Tel-Test Inc., Friendswood, TX). Construction of cDNA libraries and sequencing was performed as previously reported (Li et al., 2017). Libraries were constructed with an Illumina TruSeq Stranded mRNA kit (Illumina, San Diego, CA) and integrity was quantified using an Agilent 2100 Bioanalyzer. Libraries were sequenced using 50bp paired-end reads on an Illumina HiSEq. 2000.

$16 S$ rDNA Sequencing. The sequencing of the LIC microbial DNA from CV mice ( $n=3$ /group) was previously reported (Li et al., 2018). Briefly, DNA from the V4 region of the 16S rRNA gene was amplified and 250-bp paired-end sequencing was performed on a HiSEq. 2500 sequencer.

Serum Preparation for Aqueous Metabolite Measurements. Frozen serum samples were first thawed overnight under $4^{\circ} \mathrm{C}$, and $50 \mu \mathrm{l}$ of each serum sample was placed in a 2-ml Eppendorf vial. The initial step for protein precipitation and metabolite extraction was performed by adding $250 \mu \mathrm{l}$ of $\mathrm{MeOH}$. The mixture was then vortexed for 10 seconds and stored at $-20^{\circ} \mathrm{C}$ for 30 minutes, followed by centrifugation at $14,000 \mathrm{rpm}$ for 10 minutes at $4^{\circ} \mathrm{C}$. The supernatants $(200 \mu \mathrm{l})$ were collected into a new Eppendorf vial and dried under vacuum using an Eppendorf Vacufuge (Eppendorf, Hauppauge, NY). The dried samples were 


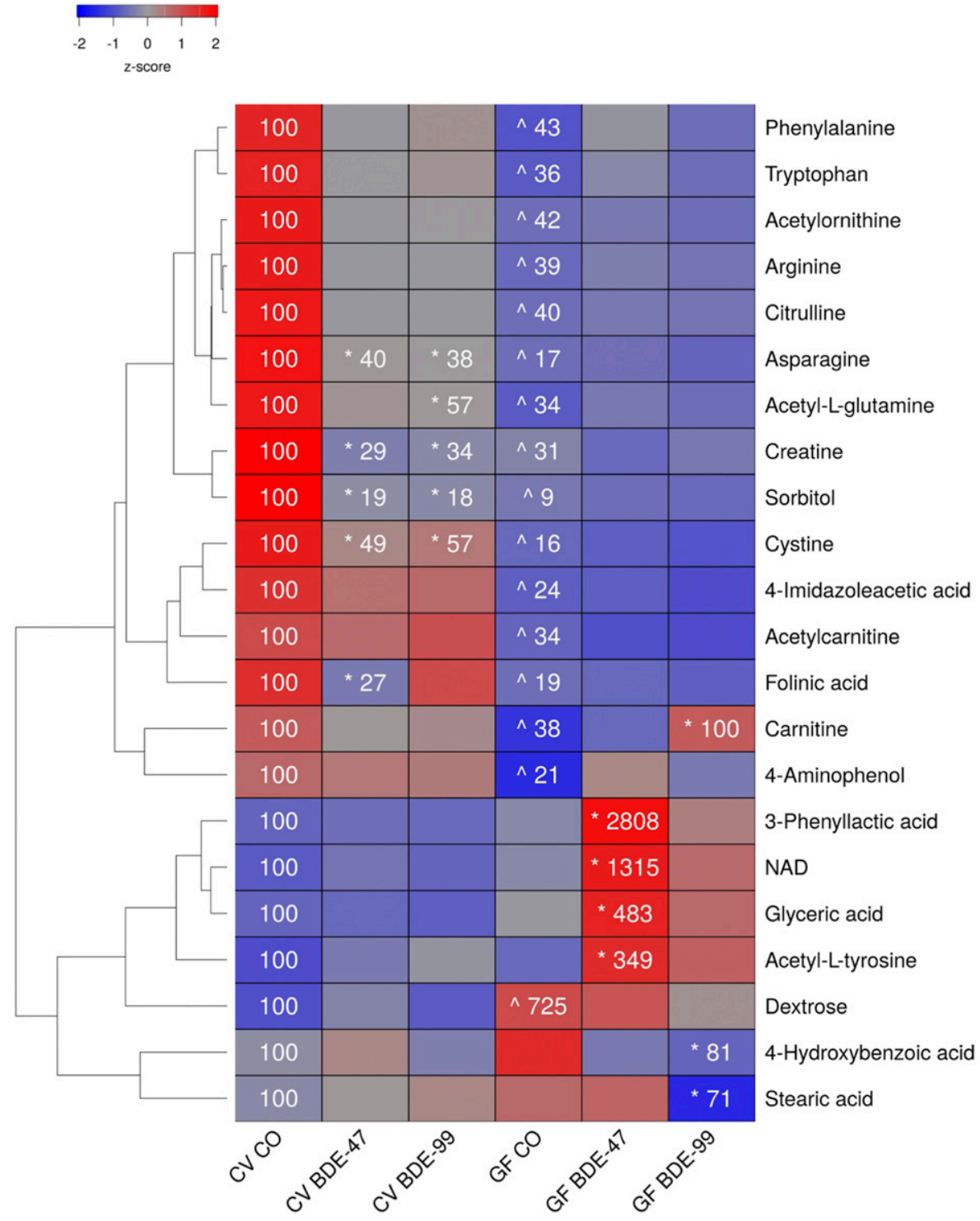

Fig. 3. Two-way hierarchical clustering dendrogram of aqueous metabolites measured using LC-MS that were differentially regulated across treatment groups in SIC of $\mathrm{GF}$ and $\mathrm{CV}$ mice following exposures to BDE-47 and BDE-99. Euclidian distance and complete linkage were used to generate the dendrogram. Red indicates higher standardized treatment group mean metabolite relative abundances ( $z$-scores) and blue lower. Asterisks (*) represent statistically significant differences between corn oil-treated and PBDE-treated groups within enterotype (adjusted $P$ value $<0.05$, Tukey's HSD post-hoc test). Caret signs $(\wedge)$ represent statistically significant baseline differences between $\mathrm{CV}$ and $\mathrm{GF}$ mice. Percent change from CVCO are shown for treatment groups with significant changes.

reconstituted in $600 \mu \mathrm{l}$ of $40 \%$ solvent $\mathrm{A} / 60 \%$ solvent B (see details in the LC-MS method section).

Tissue Preparation for Aqueous Metabolite Quantifications. The sample preparation is the same for liver, as well as small and large intestinal contents. Briefly, each tissue sample (approx. $10 \mathrm{mg}$ ) was homogenized in $200 \mu \mathrm{l}$ water in an Eppendorf tube using a Bullet Blender homogenizer (Next Advance, Averill Park, NY). Then $800 \mu \mathrm{l}$ of $\mathrm{MeOH}$ was added, and after vortexing for 10 second, the samples were stored on dry ice for 30 minutes. The samples were then sonicated in an ice bath for 10 minutes. The samples were centrifuged at $14,000 \mathrm{rpm}$ for 10 minutes $\left(4^{\circ} \mathrm{C}\right)$, and $800 \mu \mathrm{l}$ of supernatant was transferred to a new Eppendorf tube. The samples were then dried under vacuum using an Eppendorf Vacufuge (Eppendorf). Prior to MS analysis, the residue obtained was reconstituted in $600 \mu \mathrm{l}$ of $40 \%$ solvent $\mathrm{A} / 60 \%$ solvent B (see details in the LC-MS methods section next).

Measurements of Aqueous Metabolites Using LC-MS. A robust targeted liquid chromatography-tandem mass spectrometry (LC-MS/MS) method has been developed (Zhu et al., 2014) and used in a growing number of studies (Barton et al., 2015; Carroll et al., 2015; Gu et al., 2015, 2016; Reyes et al., 2015; Sood et al., 2015; Sperber et al., 2015; Zhu et al., 2015; Deng et al., 2016). Briefly, the LC-MS/MS experiments were performed on a Waters Acquity I-Class UPLC TQS-micro MS (Waters, Milford, MA) system. Each prepared serum or tissue sample was injected twice with 2 and $5 \mu \mathrm{l}$ for analysis using positive and negative ionization mode, respectively. Chromatographic separation was performed on a Waters Xbridge BEH Amide column $(2.5 \mu \mathrm{m}, 2.1 \times 150 \mathrm{~mm})$ at $40^{\circ} \mathrm{C}$. The flow rate was $0.3 \mathrm{ml} / \mathrm{min}$. For positive mode, the mobile phase was composed of solvent $\mathrm{A}$ ( $5 \mathrm{mM}$ ammonium acetate in $\mathrm{H}_{2} \mathrm{O}$ with $0.1 \%$ formic acid) and solvent $\mathrm{B}$ (ACN with $0.1 \%$ acetic acid). For negative mode, solvent $\mathrm{A}$ was $10 \mathrm{mM}$ ammonium bicarbonate in $\mathrm{H}_{2} \mathrm{O}$, and solvent $\mathrm{B}$ was $\mathrm{ACN}$. The LC gradient conditions were the same for both positive and negative ionization modes. After an initial 1.5-minute isocratic elution of $10 \% \mathrm{~A}$, the percentage of solvent $\mathrm{A}$ was increased to $65 \%$ at $t=9$ minutes. The percentage of A was then kept the same $(65 \%)$ for 5 minutes ( $t=14$ minute), and the percentage of A was decreased back to $10 \%$ at $t=15$ minutes to prepare for the next injection. The total experimental time for each injection was 30 minutes. The metabolite identities were confirmed by spiking mixtures of standard compounds. The extracted peaks were integrated using the TargetLynx software (Waters). A list of all metabolites detected across biocompartments can be found in Supplemental Table 1. Supplemental Tables 2-5 show mean abundance values and S.E. for all metabolites by exposure group that were detectable in each biocompartment.

\section{Data Analysis}

RNA-Seq. Preliminary analyses of the FASTQ files used in this study, which were deposited by our group in NCBI's Gene Expression Omnibus (GEO) (GEO accession number GSE101650) during publication of a previous manuscript, have been previously reported (Li et al., 2017). Abundance was expressed as fragments 


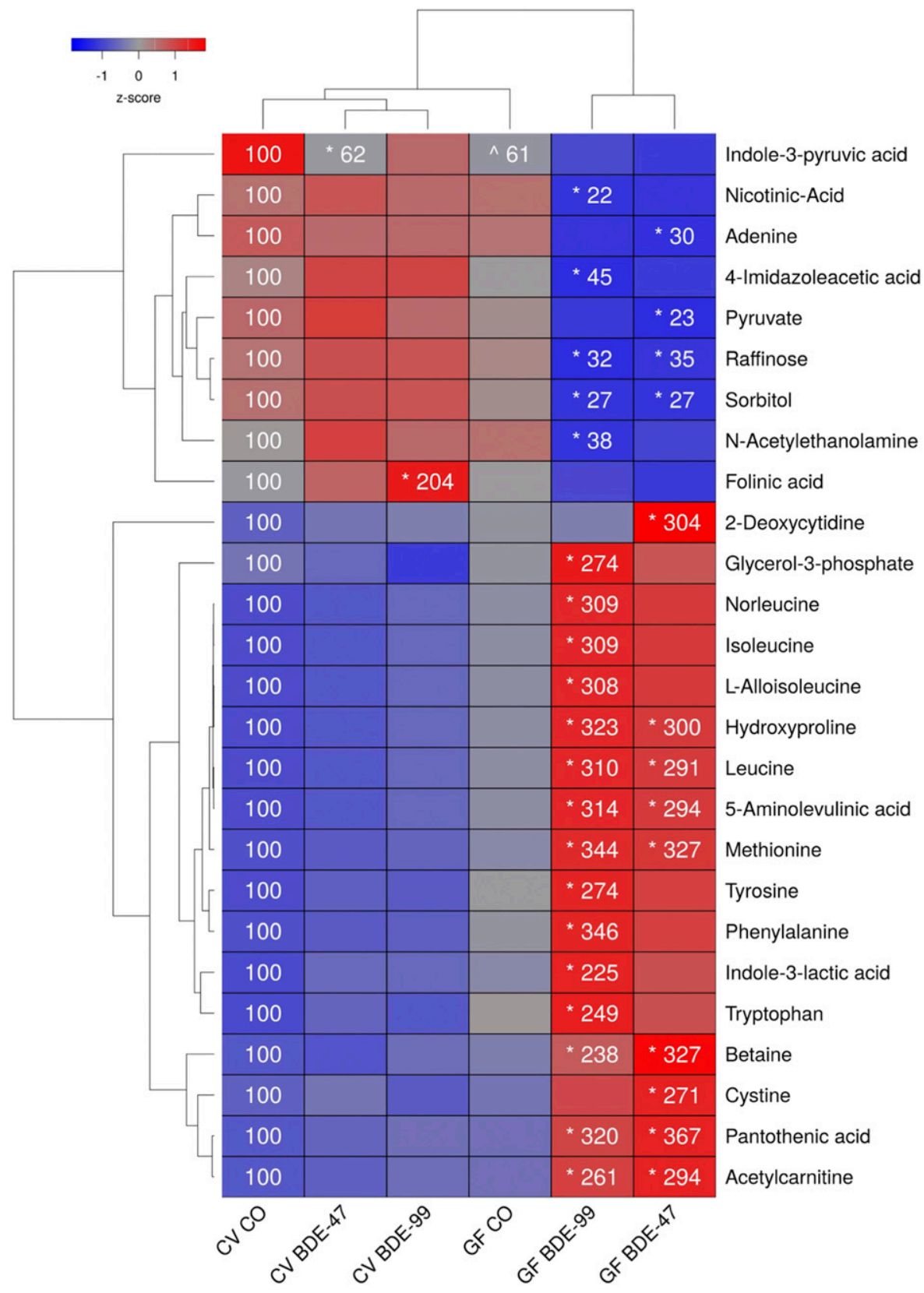

Fig. 4. Two-way hierarchical clustering dendrogram of aqueous metabolites measured using LC-MS that were differentially regulated across treatment groups in LIC of $\mathrm{GF}$ and $\mathrm{CV}$ mice following exposures to BDE-47 and BDE-99. Euclidian distance and complete linkage were used to generate the dendrogram. Red indicates higher standardized treatment group mean metabolite relative abundances ( $z$-scores) and blue lower. Asterisks (*) represent statistically significant differences between corn oil-treated and PBDE treated groups within enterotype (adjusted $P$ value $<0.05$, Tukey's HSD post-hoc test). Caret signs $(\wedge)$ represent statistically significant baseline differences between $\mathrm{CV}$ and $\mathrm{GF}$ mice. Percent change from CVCO are shown for treatment groups with significant changes.

Methionin

Tyrosine

Phenylalanin

Indole-3-lactic acid

Tryptophan

Betaine

Cystine

Pantothenic acid

Acetylcarnitine

per kilobase of transcript per million mapped reads. Differential expression analysis was performed using Cuffdiff (Trapnell et al., 2010). The differentially expressed genes were defined as false discovery rate (FDR) adjusted $P$-value $<$ 0.05 , in at least one of the PBDE-exposed groups compared with the vehicleexposed group of the same enterotype, which in this study refers to $\mathrm{CV}$ as opposed to GF mice, as well as genes that were differentially expressed by the lack of intestinal microbiome under basal conditions. Assignment of genes into intermediary metabolism categories was performed using Kyoto Encyclopedia of Genes and Genomes (KEGG) metabolism pathways in R 3.4 .3 (Kanehisa and Goto, 2000; Kanehisa et al., 2016; Kanehisa et al., 2017; R Core Team, 2017). Mean FPKM S.E. and metabolism categories for intermediary metabolism genes for each treatment group can be found in Supplemental Table 6. Fold changes and associated FDR adjusted $P$ values for comparisons between PBDE-treated mice and enterotype-matched vehicle controls and between CV and GF mice under basal conditions can be found in Supplemental Table 7. Heatmaps were also built in R 3.4.3 (base, gplots package) (Warnes et al., 2013; R Core Team, 2017). Because many gene products have multiple functions, many of the differentially expressed genes classified as intermediary metabolism genes here were reported as drug processing genes previously, mainly cytochrome $\mathrm{P} 450$ s by $\mathrm{Li}$ et al. (2017).

Aqueous Metabolites. Relative abundance values for each metabolite in each biocompartment were compared using two-way analysis of variance (ANOVA) followed by Tukey's post-hoc test using R 3.4 .3 (base, lsmeans, and multcomp packages) (Hothorn et al., 2008; Lenth, 2016; R Core Team, 2017). Heatmaps were also built in R (base, gplots package) (Warnes et al., 2013; R Core Team, 2017). Gene-metabolite network analyses were performed using MetaboAnalyst (Chong et al., 2018) (http://www.metaboanalyst.ca) using all differentially regulated intermediary metabolism genes with a fold-change $>2$ (implemented to emphasize most highly changed genes) and significantly altered metabolites in the liver. Pearson's correlations for gene and metabolite network members were calculated using R 3.4.3 (base, corrplot, and Hmisc packages) (Harrell and Dupont, 2016; Wei and Simko, 2016, R Core Team, 2017). Significant genemetabolite pair correlation plots and bar plots were created using R3 .4.3 (base, ggplot2 package) (Wickham, 2009; R Core Team, 2017)

16S rDNA Sequencing. The $16 \mathrm{~S}$ rDNA sequencing data were analyzed using QIIME v1.9.1 as previously reported (Li et al., 2018). Relative abundance was 
A

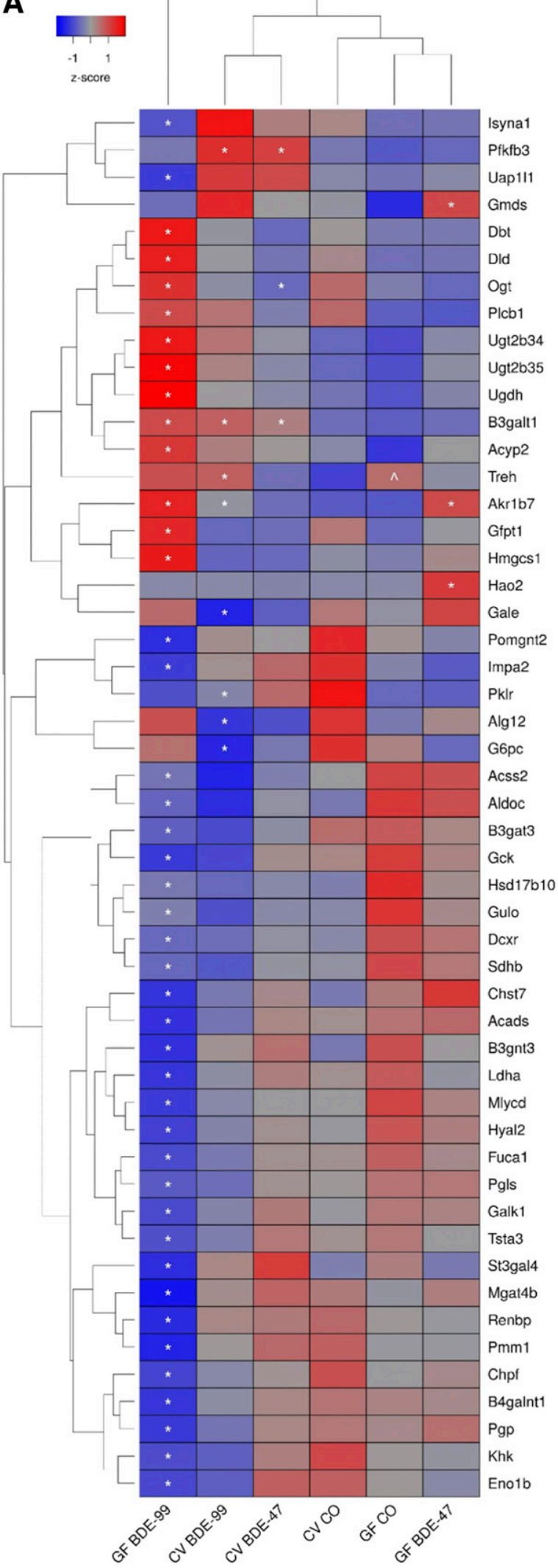

B

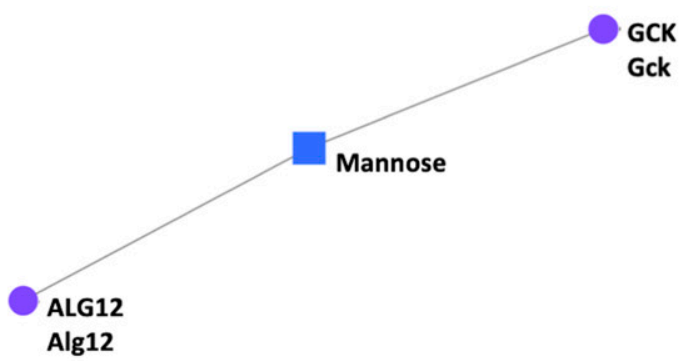

C

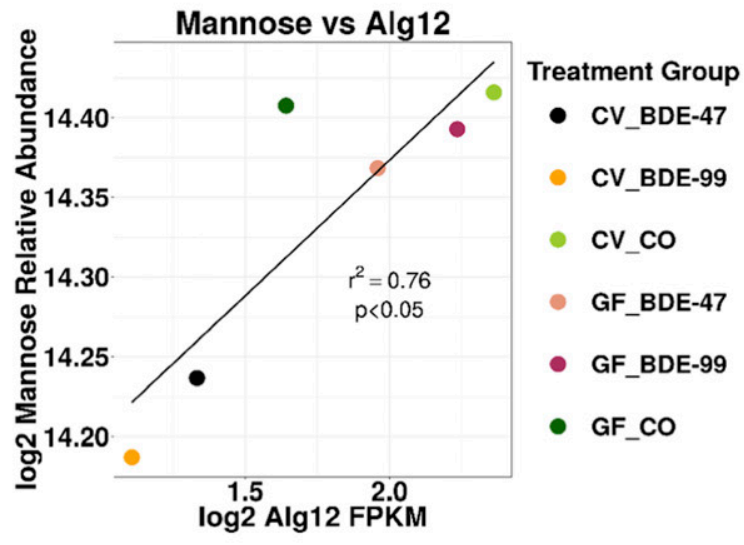

D
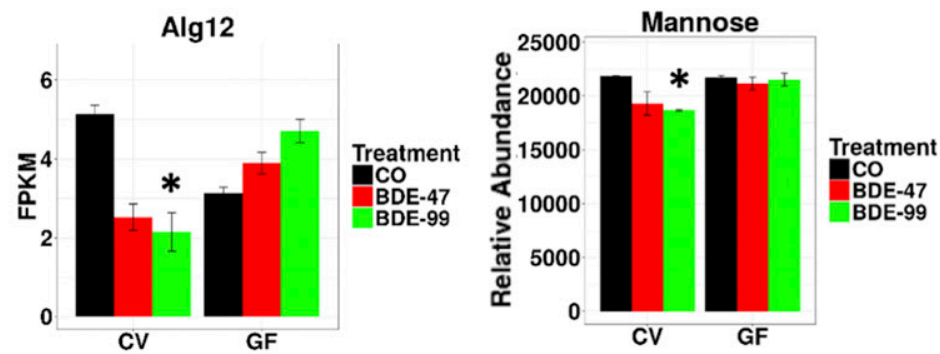

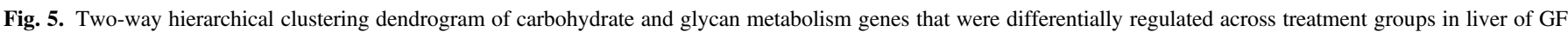

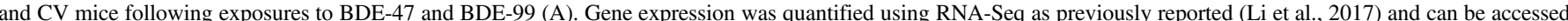

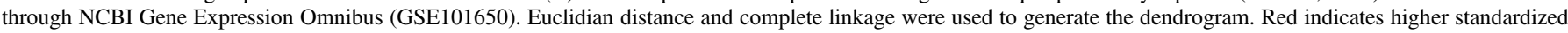

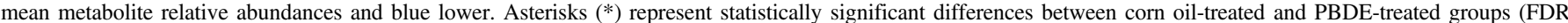

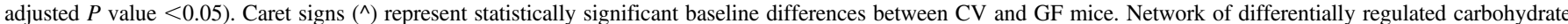

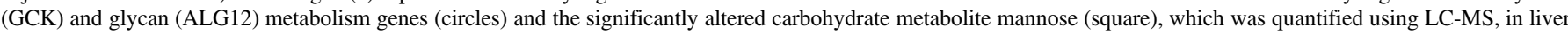


expressed as percentage of operational taxonomical units (\% OTUs) and was examined for PBDE treatment effects using a one-way ANOVA followed by Tukey's HSD post-hoc test in R 3.4.3 ( $P<0.05$ ) (R Core Team, 2017). In this study, these data were used for investigating potential associations between individual microbial taxa and serum aqueous metabolites. Spearman's correlations for differentially regulated microbial OTUs and serum metabolites by PBDEs were performed using R (base, corrplot package) (Wei and Simko, 2016; R Core Team, 2017). Significant gene-metabolite pair correlation plots and bar plots were created using R (base, ggplot2 package) (Wickham, 2009).

Glutathione. Levels of total glutathione in liver were quantified using a fluorescence-based microplate assay, as previously described (White et al., 2003; McConnachie et al., 2007; Weldy et al., 2011; Scoville et al., 2019).

Quantitative Reverse Transcription-Polymerase Chain Reaction. Expression of $\alpha$-1,6-mannosyltransferase (Alg12), glutamate-cysteine ligase catalytic subunit (GCLC), and $\mathrm{NAD}(\mathrm{P}) \mathrm{H}$ dehydrogenase (Nqo1) were measured using quantitative reverse transcription-polymerase chain reaction (RT-qPCR). Total RNA was reverse transcribed to cDNA using the High Capacity cDNA Reverse Transcription Kit (Applied Biosystems, Foster City, CA). The cDNAs were then amplified using Power SYBR Green PCR Master Mix (Applied Biosystems) using a BioRad CFX384 Real-TimePCR Detection System (BioRad, Hercules, CA). Primers were made by Integrated DNA Technologies (Coralville, IA) and primer sequences can be found in Supplemental Table 8.

\section{Results}

Effects of PBDEs on Aqueous Metabolites in Serum of CV and GF Mice. To determine the impact of PBDE exposure on the dynamic systems of intermediary metabolism, aqueous metabolites from several biocompartments were quantified using LC-MS. Of the total 169 aqueous metabolites, 139 were detectable in serum (Supplemental Tables 1 and 2). As shown in Fig. 1, the relative abundances of 13 metabolites in serum were decreased and two increased by lack of intestinal microbiome, PBDE exposure, or both, compared with CV control mice.

Six metabolites were decreased in the serum of GF mice under basal conditions, including the tryptophan metabolites 3-indoxylsulfate, 3indolepropionic acid (3-IPA), the phenylalanine metabolites phenylpyruvic acid and 3-phenyllactic acid, the branched short-chain fatty acid isobutyric acid, and the fatty acid amide $N$-acetylethanolamine. Acetylglycine and the saturated long-chain fatty acid palmitic acid were increased in serum under basal conditions in GF mice.

PBDEs differentially regulated 12 serum metabolites. Interestingly, the relative abundances of three tryptophan metabolites, namely the microbial metabolites 3-IPA and indole-3-acetic acid, as well as the host metabolite kynurenine, were all decreased by both PBDE congeners in $\mathrm{CV}$ but not GF mice. PBDEs also decreased $N$-acetylethanolamine and phenyllactic acid in CV but not GF mice. BDE-47 decreased the relative abundances of the amino acid valine and its nonproteogenic isomer norvaline, whereas BDE-99 increased phenylpyruvic acid, isobutyric acid, and the carbohydrate metabolite sorbitol also in CV but not GF mice. In contrast, the lack of gut microbiota predisposed GF mice to PBDE-mediated decreases in the relative abundance of metabolites derived from the branched-chain amino acid (BCAA) valine. BDE-47 increased $\alpha$-ketoisovaleric, whereas both BDE-47 and BDE-99 mediated increased $\alpha$-hydroxyisovaleric acid.

Effects of PBDEs on Aqueous Metabolites in Liver of CV and GF Mice. In liver, 156 aqueous metabolites were detectable (Supplemental Table 3). As shown in Fig. 2, under basal conditions, 11 out of the 16 metabolite changes occurred between CV and GF mice.
The relative abundances of the sialic acid $N$-acetylneuraminic acid, the purine metabolite urate, and the amino acid glycine were all increased in GF mice. The relative abundances of isobutyric acid, 3-phenyllactic acid, the amino acid derivative carnitine, the amino acid derivative ketoleucine, the carbohydrate metabolite glucuronic acid, and dietderived metabolites (benzoic acid, phthalic acid, 4-methoxyphenylacetic acid) were all decreased in GF mice.

In $\mathrm{CV}$ mice, BDE-47 had minimal effect on the hepatic aqueous metabolite profiles. However, BDE-99 decreased relative abundances of carnitine, the carbohydrate mannose, the antioxidant glutathione, the homocysteine metabolite cystathionine, and the vitamin nicotinamide, all in CV but not GF mice. The lack of gut microbiota predisposed GF mice to BDE-47-mediated decreases in the glutamine derivative acetylL-glutamine.

Effects of PBDEs on Aqueous Metabolites in SIC of CV and GF Mice. A total of 147 aqueous metabolites were detectable in the SIC (Supplemental Table 4). As shown in Fig. 3, there were 15 metabolites that were decreased in SIC of GF mice under basal conditions, including several amino acids (phenylalanine, tryptophan, arginine, and cysteine, citrulline, asparagine), amino acid derivatives (carnitine, acetyl-L-glutamine, creatine, acetylornithine, and 4-imidazoleacetic acid), the carbohydrate metabolite sorbitol, as well as other organic compounds (folinic acid, and 4-aminophenol). Conversely, the relative abundance of dextrose (also known as D-glucose) was increased in the SIC of GF mice under basal conditions.

PBDE exposure differentially regulated six metabolites in the SIC of $\mathrm{CV}$ mice but not GF mice. Asparagine, creatine, sorbitol, and cysteine were decreased by both PBDE congeners, folinic acid was decreased by BDE-47, and acetyl-L-glutamine was decreased by BDE-99. In contrast, the lack of gut microbiota predisposed GF mice to BDE-47-mediated increases in the relative abundances of amino acid derivatives (3phenyllactic acid, acetyl-L-tyrosine), the redox active coenzyme NAD, and the glycerol derivative glyceric acid; BDE-99 mediated an increase in carnitine; and BDE-99 mediated a decrease in 4-hydroxybenzoic acid.

Effects of PBDEs on Aqueous Metabolites in LIC of CV and GF Mice. A total of 147 aqueous metabolites were detectable in the SIC (Supplemental Table 5). As shown in Fig. 4, PBDEs differentially regulated 26 metabolites in the LIC - the highest among the four biocompartments examined. Interestingly, the relative abundances for the majority of metabolites (24 out of 26) in the LIC were changed by PBDEs only in GF conditions but not in CV conditions. The two exceptions are the tryptophan metabolite indole-3-pyruvic acid, which was reduced in the LIC of CV mice by BDE-47 (the basal relative abundance in GF mice was also lower), and folinic acid, which was increased by BDE-99 in CV but not GF mice.

The lack of gut microbiome predisposed GF mice to PBDE-mediated increases in 17 LIC metabolites. Compared with vehicle-exposed GF mice, both BDE-47 and BDE-99 increased the relative abundances of the amino acids leucine and methionine, amino acid derivatives (4hydroxyproline, 5-aminoleulinic acid, and acetylcarnitine), the choline metabolism intermediate and methionine precursor betaine, and the coenzyme A precursor pantothenic acid. BDE-47 increased relative abundances of the nucleic acid 2-deoxycytidine and amino cysteine in LIC of GF mice, whereas BDE-99 increased several amino acids (norleucine, isoleucine, L-alloisoleucine, tyrosine, phenylalanine, and tryptophan), the tryptophan metabolite indole-3-lactic acid, and the carbohydrate metabolite glycerol-3-phosphate.

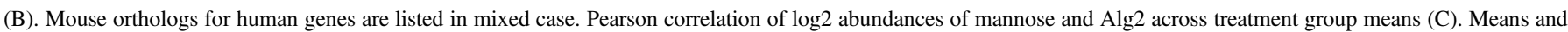

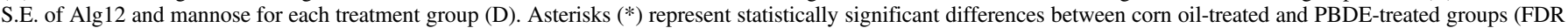
adjusted $P$ value $<0.05$, Cuffdiff). Caret signs $(\wedge)$ represent statistically significant baseline differences between $\mathrm{CV}$ and GF mice. 


\section{A Carbohydrate and Glycan Metabolism}

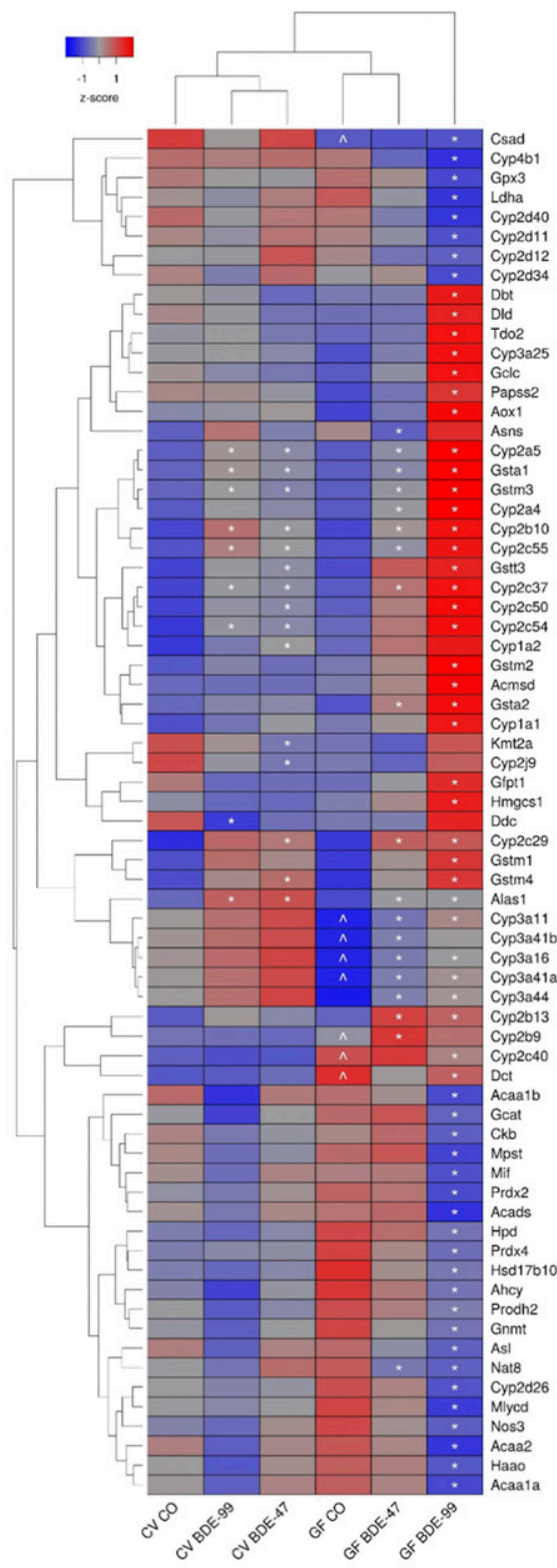

B

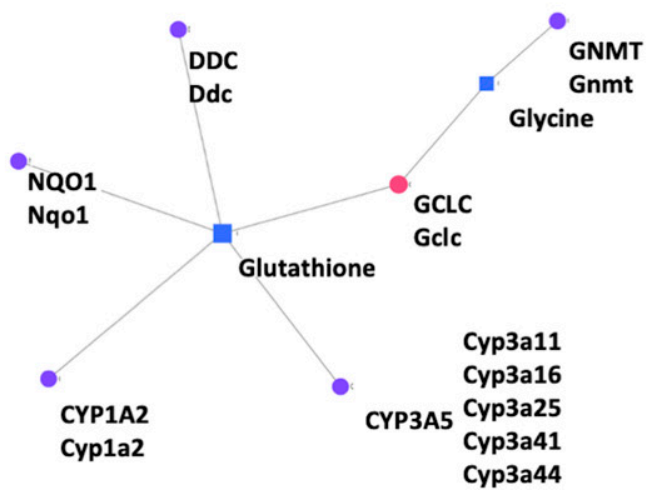

\section{Vitamin and Cofactor Metabolism}

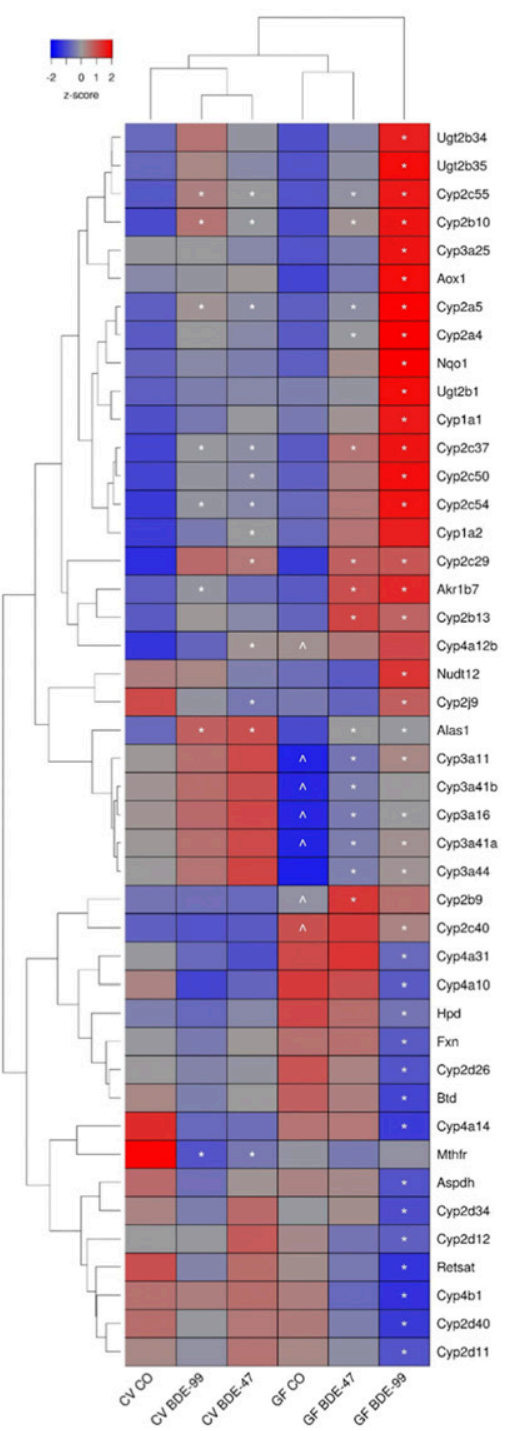

CYP3A4

Cyp3a11

Cyp3a16

Cyp3a25

Cyp3a41

Сур3а44

Fig. 6. Two-way hierarchical clustering dendrogram of amino acid-metabolism genes that were differentially regulated across treatment groups in liver of GF and CV mice following exposures to BDE-47 and BDE-99 (A). Gene expression was quantified using RNA-Seq as previously reported (Li et al., 2017) and can be accessed through NCBI Gene Expression Omnibus (GEO; GSE101650). Euclidian distance and complete linkage were used to generate the dendrogram. Red indicates higher standardized mean metabolite relative abundances and blue lower. Network of differentially regulated amino acid metabolism genes [dopa decarboxylase (DDC), NQO1, GNMT, CYP1A2, and CYP3A5] (circles) and significantly altered aqueous metabolites (glycine and glutathione) (squares), which were measured using LC-MS, in liver (B). Mouse orthologs for 
The lack of gut microbiota also predisposed GF mice to PBDEmediated decreases in seven metabolites. Both BDE-47 and BDE-99 decreased the relative abundances of carbohydrate metabolites sorbitol and raffinose in the LIC of GF mice compared with control GF mice. In addition, BDE-47 decreased relative abundances of the nucleic acid adenine and carbohydrate metabolite pyruvate, whereas BDE-99 decreased the NAD precursor nicotinic acid, the histamine metabolite 4imidazoleacetic acid, and the fatty acid amide $N$-acetylethanolamine in the LIC of GF mice.

Differentially abundant metabolites unique and common to different biocompartments are summarized in Supplemental Fig. 4. Treatment group-specific and common differentially abundant metabolites within each biocompartment are summarized in Supplemental Fig. 5.

Liver Intermediary-Metabolism-Gene Expression and Gene-Metabolite Networks. As shown in Figs. 5 and 6, 133 intermediary metabolism-related genes belonging to one or more of the KEGG pathways (carbohydrate, glycan, amino acid, vitamin and cofactor, nucleotide, and energy metabolism) were differentially expressed owing to either PBDE exposure, lack of gut microbiota, or both in liver. Specifically, carbohydrate metabolism and glycan biosynthesis and metabolism were represented by 38 and 13 differentially expressed genes, respectively (Fig. 5A). Amino acid metabolism was represented by 70 differentially expressed genes (Fig. 6A) and vitamin and cofactor metabolism contained 44 genes (Fig. 6C). Nucleotide metabolism and energy metabolism contained 11 and 10 differentially expressed genes, respectively (Supplemental Fig. 1). Metabolism category labels for genes in Fig. 5A, Fig. 6, A and C, and Supplemental Fig. 1 can be found in Supplemental Table 6.

To integrate the liver intermediary metabolism transcriptomic and metabolomic data and identify potential gene-metabolite regulatory networks impacted by PBDEs, network analyses were performed using MetaboAnalyst of all differentially regulated intermediary metabolismrelated genes and aqueous metabolites in liver. As MetaboAnalyst was built for human genes, Ensembl Biomart was used to retrieve human homologs.

The Network Explorer module revealed three networks using the list of differentially regulated liver genes and metabolites. One of the networks generated by MetaboAnalyst centered on the carbohydrate metabolite mannose with connections (representing interactions from literature found by Network Explorer) to the carbohydrate metabolism gene glucokinase (GCK), the glycan synthesis gene ALG12, and the carbohydrate mannose (Fig. 5B). In addition, a significant correlation was observed in our data between ALG12 expression and the relative abundance of mannose (Figs. 5C), and BDE-99 decreased both in CV but not GF mice (Fig. 5D). Expression of Alg12 measured using RTqPCR matched RNA-seq expression patterns across treatment groups (Supplemental Fig. 2).

The second gene-metabolite network that was generated by MetaboAnalyst centered around glutathione (Fig. 6B), which is synthesized from three amino acids (glycine, glutamate, and cysteine). Glutathione was connected to the amino acid metabolism genes dopa decarboxylase (DDC), cytochrome P450 1A2 (CYP1A2 representing the mouse Cyp1a2), CYP3A5 (representing several mouse Cyp3a isomers), and
GCLC (representing the mouse Gclc), which is a glutathione-synthesis gene utilizing glutamate and cysteine as substrates (Fig. 6B). Expression of Cyp1a2 and Cyp3a11, which was the most highly expressed Cyp3a isoform, have been validated using RT-qPCR as previously reported ( $\mathrm{Li}$ et al., 2017). NAD(P)H dehydrogenase, quinone 1 (NQO1), which is classified as a vitamin and cofactor metabolism gene as well as a prototypical target gene of the antioxidative stress sensor nuclear factor erythroid 2-like 2 (Nrf2), is also connected to glutathione (Fig. 6, B and C) (Ross and Siegel, 2018). Levels of liver glutathione were also measured independently using a fluorescence-based microplate assay. Interestingly, the pattern across exposure groups was similar to that found using the LC-MS-based aqueous metabolite panel, suggesting that although the transcriptomic changes in glutathione synthesis/ metabolism-related genes occur following PBDE exposure and the absence of gut microbiome, the liver serves as an efficient repertoire to buffer the oxidative stress by maintaining the total glutathione levels (Supplemental Fig. 3, A and B). To validate the RNA-Seq results, RTqPCR was performed for the mRNA expression of Gclc and Nqo1, and the expression patterns were consistent among the two assays across exposure groups (Supplemental Fig. 3, C-F).

The third network generated was centered on the vitamin nicotinamide, which was connected to vitamin- and cofactor-processing genes CYP2D6 and CYP3A5, represented by several mouse Cyp2d and Cyp3a isoforms, respectively (Fig. 6D).

Correlations between Serum Metabolites and Gut Microbiota. To investigate the intersection of PBDE regulation of serum metabolites and the gut microbiome at the individual taxa level, the relative abundances of 23 gut microbial taxa that were changed by PBDEs were tested for correlation with PBDE-regulated serum metabolites. Serum metabolites were prioritized because the changes in this biocompartment could have systemic effects. Of the 23 taxa, 19 were significantly correlated with at least one serum aqueous metabolite (Fig. 7).

Regarding amino acid metabolism, the microbial tryptophan metabolite 3-IPA was positively correlated with the largest number of taxa (8), all of which belong to the phylum Firmicutes, and apart from one taxon from the Cloistridia class, all belong to the class Bacilli (Fig. 7). Four of the Bacilli belong to the Bacillales order and three are Lactobacillales. Within the Bacillales order, two taxa were in the Staphylococcaceaes family, one is a Baciliaceae, and the other is unresolved at the family level and beyond. Both Staphylococcaceaes are Staphylococcus at the genus level, but neither is resolved at the species level. The taxon from the Baciliaceae family is Bacillus at the genus level and also unresolved at the species level. Within the Lactobacillale order, two are in the Lactobacillaceae family, and the other taxon is unresolved at the family level and beyond. Both Lactobacillaceae are Lactobacillus at the genus level. One is the reuteri species and the other is unresolved at the species level. The taxon in the Clostridia class is Clostridiales (order), Lachnospiraceae (family), and unresolved at the genus and species levels.

Another microbial tryptophan metabolite, namely indole-3-acetic acid, is positively correlated with two microbial taxa and inversely correlated with one (Fig. 7). The first positively correlated taxon is Firmicutes (phylum), Clostridia (class), Clostridiales (order),

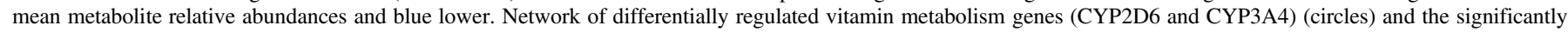

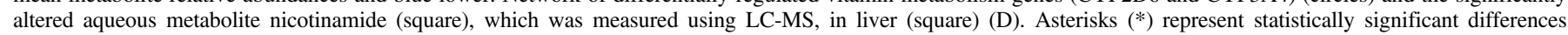

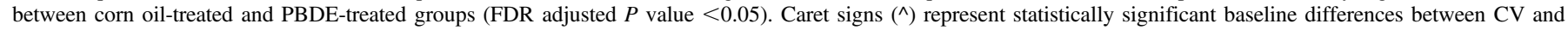
GF mice. 


\section{Serum Aqueous Metabolites vs LIC Microbiome Taxa}

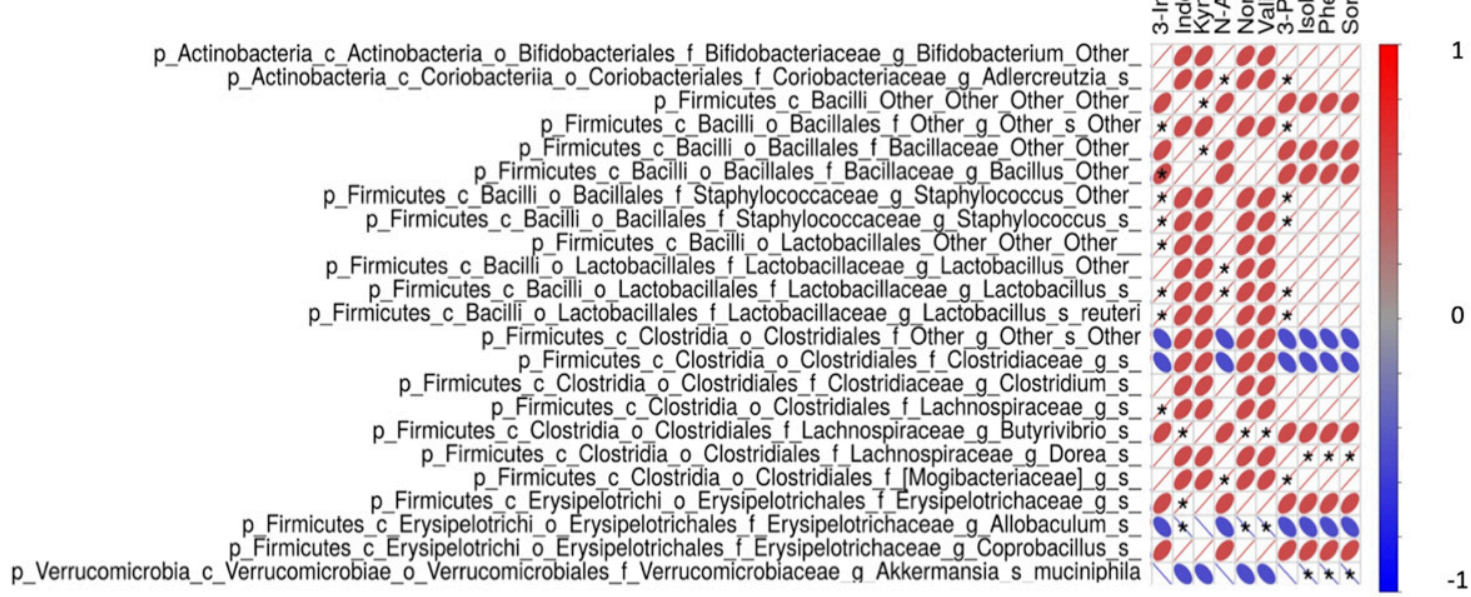

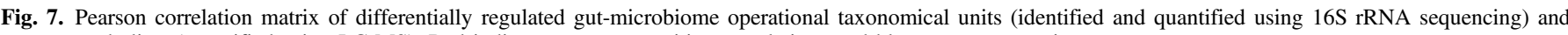
serum metabolites (quantified using LC-MS). Red indicates stronger positive correlations and blue stronger negative.

Lachnospiraceae (family), Butyrivibrio (genus), and unresolved at the species level. The second positively correlated taxon is Firmicutes (phylum), Erysipelotrichi (class), Erysipelotrichales (order), Erysipelotrichaceae (family), and unresolved at the genus and species level. The inversely correlated taxon has the same taxonomy as the Erysipelotrichaceae (family), belongs to the Allobaculum genus, and is unresolved at the species level.

The phenylalanine metabolite phenyllactic acid is positively correlated with seven gut microbial taxa (Fig. 7). Two of the seven taxa are the same Staphyllococcus genus as described above. An additional two are the same Lactobacillus genus taxa, one identified as reuteri, and the other unresolved at the species level. The fifth taxon associated with phenyllactic acid is Bacillales at the order level and is also previously described above. One of the remaining taxa associated with phenyllactic acid is Actinobacteria (phylum), Coriobacteriia (class), Coriobacteriales (order), Coriobacteriaceae (family), Adlercreutzia (genus), and unresolved at the species level. The other taxon is Firmicutes (phylum), Clostridia (class), Clostridiales (order), Mogibacteriaceae (family), and unresolved at the genus and species levels.

BCAAs valine and norvaline are positively correlated with the same taxon from the Butyrivibrio genus as indole-3-acetic acid (Fig. 7). They are both also inversely correlated with the same taxon from the Allobaculum genus as indole-3-acetic acid (Fig. 7).

The fatty acid amide $N$-acetylethanolamine is positively correlated with four microbial taxa (Fig. 7). The full taxonomy of three have already been described: Adlercreutzia (genus), Lactobacillus (genus, unresolved at species level), and Mogibacteriaceae (family). The fourth taxa associated with $N$-acetylethanolamine has the same taxonomy as the Lactobacillus (genus), and also unresolved at the species level, but is distinct from the Lactobacillus (genus) associated with 3-IPA and 3phenyllactic acid.

Three metabolites, namely the branched short-chain fatty acid isobutyric acid, the phenylalanine metabolite and phenyllactic acid precursor phenylpyruvic acid, and the carbohydrate sorbitol, are all positively correlated with a microbial taxon that is Firmicutes (phylum), Clostridia (class), Clostridiales (order), Lachnospiraceae (family), Dorea (genus), and unresolved at the species level (Fig. 7). These same three metabolites are also inversely associated with Akkermansia (genus) mucinophila (species), which belongs to Verrucomicrobia phylum, Verrucomicrobiae class, Verrucomicrobiales (order), and Verrucomicrobiaceae family.

\section{Discussion}

Although the commercial pentaBDE (composed mainly of BDE-47 and BDE-99) and octaBDE formulations of PBDEs were voluntarily taken off the market in 2004 and decaBDE by 2014 (2017), PBDEs are still readily detectable in human samples. Although concentrations of pentaBDE constituents have previously been observed to decline since their withdrawal, the trend may have flattened out and concentrations may now be on the rise (Ma et al., 2013; Zota et al., 2013; Hurley et al., 2017; Parry et al., 2018; Cowell et al., 2019). This is probably the result of the persistent and bioaccumulative nature of PBDEs, the recycling of PBDE-containing products worldwide, and the potential for more highly brominated PBDEs to degrade in the environment into lower brominated forms (Bezares-Cruz et al., 2004; Zeng et al., 2008).

As originally reported in $\mathrm{Li}$ et al. (2017), male mice were dosed once daily for four consecutive days with $48.5 \mathrm{mg} / \mathrm{kg} \mathrm{BDE}-47$ and $56.5 \mathrm{mg} / \mathrm{kg}$ BDE-99 $(100 \mu \mathrm{mol} / \mathrm{kg})$. Although these doses are high compared with estimated human PBDE exposures, they were selected to allow for comparison of acute xenobiotic metabolism gene expression changes with previously published studies (Pacyniak et al., 2007; Sueyoshi et al., 2014; Li et al., 2017). In this study, metabolomic data from archived samples collected by $\mathrm{Li}$ et al. was integrated with previously reported liver gene expression ( $\mathrm{Li}$ et al., 2017) and intestinal microbiome data ( $\mathrm{Li}$ et al., 2018) to explore acute PBDE effects on intermediary metabolism.

Limitations of this study include sample size and the evaluation of only male mice only, and that it was mainly associative, all of which stem from the use of archived samples and previously reported datasets. As reported in Li et al. (2017), male mice were used exclusively owing to changes in hormone levels during the estrous cycle. Such hormonal variation is also relevant to this study as it could independently impact glucose metabolism and other intermediary metabolic pathways (Mauvais-Jarvis et al., 2013). Sample size for the three data sets used 
in this study was also a limitation. However, even with relatively small sample sizes, we were able to identify statistically significant PBDE and gut microbiome-associated changes in intermediary metabolites, expression of associated genes, and correlations among metabolites and several previously reported human health and disease relevant microbes (Li et al., 2018). Importantly, future mechanistic studies will be needed to fully validate the observed findings of this study.

Tryptophan Pathway and Involvement of PBDEs in Metabolic Syndrome. Notably, serum 3-IPA, which is a tryptophan microbial metabolite known to be protective against diabetes and inflammation, was decreased by both PBDE congeners in CV mice but not in GF mice (Fig. 1). This highlights the potential involvement of the tryptophan metabolism pathway in PBDE-mediated metabolic disorders, because 3-IPA is produced by intestinal bacteria and accumulates in the blood to modulate systemic effects (Dodd et al., 2017) such as metabolic syndrome. Specifically, 3-IPA is associated in epidemiology studies with reduced risk of type 2 diabetes and may preserve pancreatic $\beta$ cell function (de Mello et al., 2017). These observations in humans were further supported in rats because 3-IPA improved glucose metabolism, as evidenced by reduced fasting plasma insulin and the homeostatic model assessment index of insulin resistance (Abildgaard et al., 2018). In humans, BDE-47 was associated with diabetes in two independent community-based epidemiologic studies (Zhang et al., 2016), and total PBDEs were linked to increased risk of diabetes during pregnancy (Eslami et al., 2016; Smarr et al., 2016). PBDE-exposed rats developed hyperglycemia and aberrant expression of genes involved in glucose homeostasis (Zhang et al., 2016; Krumm et al., 2018), corresponding to disturbed glucose and insulin metabolism in adipose tissue, disturbed lipid metabolism in liver, and increased ketone concentrations in blood (Cowens et al., 2015). PBDEs disturbed glucose signaling in hepatocytes (Søfteland et al., 2011). In vitro studies showed that PBDEs disrupted insulin-producing pancreatic $\beta$ cells (Karandrea et al., 2017). Therefore, PBDE-mediated decrease in 3-IPA, by disrupting glucose and insulin signaling, may be among the toxification mechanisms for the pathogenesis of diabetes.

Another potential involvement of 3-IPA in metabolic syndrome is inflammation - an important contributor to diabetes (Donath and Shoe1son, 2011; Goldfine et al., 2011)_as 3-IPA is known to suppress inflammatory genes by antagonizing aryl hydrocarbon receptor functions (Yisireyili et al., 2017). In addition, 3-IPA is a pregnane $\mathrm{X}$ receptor (PXR) ligand in intestine, and activation of PXR reduces intestinal inflammation (Cheng et al., 2012; Venkatesh et al., 2014). Conversely, PBDEs have been shown to affect the immune system during development (Zhou et al., 2006; Martin et al., 2007; Liu et al., 2012; Bondy et al., 2013; Koike et al., 2014; Lv et al., 2015), as well as promote inflammation and oxidative stress (Zhang et al., 2013), which are key contributors for the pathogenesis of diabetes (Farah et al., 2008; Vikram et al., 2014; Domingueti et al., 2016; Hojs et al., 2016). Therefore, a PBDE-mediated decrease in IPA may also contribute to the pathogenesis of diabetes by promoting inflammation.

Branched-Chain Amino Acid Metabolites, PBDEs, and Adipose Tissue Metabolism. Isobutyric acid is a branched short-chain fatty acid derived from the BCAA valine and can be produced by gut bacteria or by host (Liebich and Först, 1984; Zarling and Ruchim, 1987; Cole, 2015) . Shown to potentially benefit adipocyte metabolism, isobutyric acid was decreased in this study in GF mice under basal conditions and by BDE99 in CV mice (Heimann et al., 2016) . Although there is controversy as to a potentially beneficial or detrimental role for BCAAs in obesity and type 2 diabetes, preliminary evidence suggests a potential protective role for their metabolites(Lynch and Adams, 2014; Heimann et al., 2016) ).

In obesity, adipocytes exhibit inter-related changes, including increased basal rates of lipolysis and insulin resistance, that are thought to contribute to systemic insulin resistance (Girousse et al., 2013; Arner and Langin, 2014; Morigny et al., 2016). Treatment of rat adipocytes with isobutyric acid reduced lipolysis and improved basal and insulininduced glucose uptake (Heimann et al., 2016) (). Nonbranched shortchain fatty acids have also been observed to have similar effects on rat adipocytes (Heimann et al., 2014). In contrast, PBDEs have been associated with increased rat adipocyte lipolysis and decreased glucose oxidation (marker of uptake and metabolism) (Hoppe and Carey, 2007). Furthermore, PBDEs have been associated with insulin resistance among obese humans (Helaleh et al., 2018).

The decreases of the potentially beneficial valine metabolite isobutyric acid and, among GF mice, its upstream metabolic intermediates $\alpha$-ketoisovaleric and $\alpha$-hydroxyisovaleric acids, suggests that PBDEs may promote metabolic syndrome in part by interference with valine/ BCAA metabolism.

PBDEs, Protein Glycosylation, and Metabolic Syndrome. Liver intermediary metabolism-gene expression and aqueous metabolite data were integrated using the Network Explorer module in MetaboAnalyst (Chong et al., 2018). One of the resulting networks, consisting of the genes ALG12, GCK, and the carbohydrate mannose, stood out owing to a correlation found in the study data between Alg12 and mannose. As part of the N-glycosylation pathway, ALG12 is responsible for the addition of the eighth mannose residue to a growing oligosaccharide that ultimately possesses nine mannoses and three glucoses, all of which are added in the endoplasmic reticulum lumen (Burda and Aebi, 1999; Thiel et al., 2002; Aebi, 2013). As an N-glycan, this molecule is subsequently attached to the nitrogen of the side chain of asparagine in a protein by oligosaccharyltransferase (Burda and Aebi, 1999; Aebi, 2013). $\mathrm{N}$-glycans on proteins participate in the luminal endoplasmic reticulumassociated degradation pathway that detects and degrades misfolded proteins (Quan et al., 2008).

Epidemiologic studies have found that patients diagnosed with metabolic syndrome have an altered $\mathrm{N}$-glycan profile, with some components, including those with seven and eight mannose residues, being positively associated with signs of metabolic syndrome and some inversely (Lu et al., 2011; McLachlan et al., 2016). In this study, BDE99 reduced Alg12 expression and the relative abundance of mannose in CV but not GF mice, suggesting that a PBDE microbial metabolite could also contribute to these changes. To our knowledge, the potential for PBDEs and/or PBDE host/microbial metabolites to interfere with protein glycosylation has not been explored. However, future glycomics studies will be necessary to evaluate this potential novel mechanism of PBDE toxicity.

Microbiota Correlations with Serum Metabolites. The present study showed that PBDE-mediated decreases in Lactobacillus reuteri correlate with serum decreases in the tryptophan metabolite 3-IPA. Even though $L$. reuteri is not known to produce 3-IPA, it does produce indole3-lactic acid, which is a 3-IPA precursor (Dodd et al., 2017). PBDEs also reduced serum indole-3-acetic acid, which is another tryptophan metabolite produced by L. reuteri (Cervantes-Barragan et al., 2017). Interestingly, in mouse models into which $L$. reuteri has been introduced in combination with tryptophan supplementation, indole-3-acetic and indole-3-lactic acid were shown to promote the development of intestinal $\mathrm{T}$ cells that improve immune tolerance to antigens in the diet (Cervantes-Barragan et al., 2017). However, given the small sample size, microbe-metabolite associations should be viewed as preliminary until follow up studies with larger numbers are completed.

Summary. The present study demonstrates that oral PBDE exposure profoundly altered many important intermediary metabolites involved in amino acid, carbohydrate, and lipid metabolism, in a biocompartmentspecific manner. Although liver metabolites were influenced more by enterotype than PBDEs, metabolites from large intestinal contents were 
influenced more by PBDEs than by enterotype and such influence occurred almost exclusively in GF conditions. Metabolites from serum and SIC were regulated by both enterotype and PBDEs. Two interesting regulatory patterns were discovered: 1 ) The presence of gut microbiota is necessary for PBDE-mediated decreases in metabolites, such as those from the tryptophan pathway in serum and those from the metabolism of amino acids and carbohydrates in the SIC; 2) lack of gut microbiota predisposed mice to PBDE-mediated changes in metabolites, including the majority of those from amino acid and carbohydrate metabolism in the LIC. Correlation analyses among targeted metabolomics, hepatic RNA-Seq, and intestinal 16S rDNA sequencing further established potential mechanisms for PBDE-mediated regulation of intermediary metabolites. Together our observations highlight the potential involvement of dysregulation of intermediary metabolism as part of the mechanisms underlying the multiorgan toxicities of PBDEs and unveils the important role of gut microbiota as a key internal modifier for PBDEmediated host response. Follow-up studies will include mechanistic investigation using male and female wild-type and nuclear receptor knockout mice to assess the impact of key metabolites identified in this study on PBDE toxicity, dysbiosis, and potential interactions with nuclear receptors. Inflammation, glucose homeostasis, and the effects of high-fat diet will also be explored.

\section{Acknowledgments}

The authors thank GNAC technical support for the germ-free mouse experiment, Dr. Debbie Nickerson's laboratory at UW Genome Sciences for sequencer access and support for the A-Seq experiments, Brian High from UW DEOHS IT team for providing server access and advice to improve our A-Seq bioinformatics pipeline, the Northwest Metabolomics Research Center for targeted metabolomics, Dianne Botta and Collin White from Dr. Terrance Kavanagh's laboratory for their guidance measuring glutathione, and the members of Dr. Cui's laboratory for help in tissue collection and manuscript revision.

\section{Authorship Contributions}

Participated in research design: $\mathrm{Li}$, Cui.

Conducted experiments: Li, Dempsey, Wang, Gu, Cui.

Contributed new reagents or analytic tools: Raftery.

Performed data analysis: Scoville, Li, Wang, Gu, Cui.

Wrote or contributed to the writing of the manuscript: Scoville, Li, Dempsey, Wang, Raftery, Mani, Gu, Cui.

\section{References}

Abildgaard A, Elfving B, Hokland M, Wegener G, and Lund S (2018) The microbial metabolite indole-3-propionic acid improves glucose metabolism in rats, but does not affect behaviour. Arch Physiol Biochem 124:306-312.

Aebi M (2013) N-linked protein glycosylation in the ER. Biochim Biophys Acta 1833 (11): 2430-2437.

Arner P and Langin D (2014) Lipolysis in lipid turnover, cancer cachexia, and obesity-induced insulin resistance. Trends Endocrinol Metab 25:255-262.

Barton S, Navarro SL, Buas MF, Schwarz Y, Gu H, Djukovic D, Raftery D, Kratz M, Neuhouse $\mathrm{ML}$, and Lampe JW (2015) Targeted plunknownasma metabolome response to variations in dietary glycemic load in a randomized, controlled, crossover feeding trial in healthy adults. Food Funct 6:2949-2956.

Bezares-Cruz J, Jafvert CT, and Hua I (2004) Solar photodecomposition of decabromodiphenyl ether: products and quantum yield. Environ Sci Technol 38:4149-4156.

Bondy GS, Lefebvre DE, Aziz S, Cherry W, Coady L, Maclellan E, Armstrong C, Barker M, Cooke G, Gaertner D, et al. (2013) Toxicologic and immunologic effects of perinatal exposure to the brominated diphenyl ether (BDE) mixture DE-71 in the Sprague-Dawley rat. Environ Toxicol 28:215-228.

Burda P and Aebi M (1999) The dolichol pathway of N-linked glycosylation. Biochim Biophys Acta 1426 (2):239-257, doi: 10.1016/s0304-4165(98)00127-5 9878760.

Carroll PA, Diolaiti D, McFerrin L, Gu H, Djukovic D, Du J, Cheng PF, Anderson S, Ulrich M, Hurley JB, et al. (2015) Deregulated Myc requires MondoA/Mlx for metabolic reprogramming and tumorigenesis. Cancer Cell 27:271-285.

Cervantes-Barragan L, Chai JN, Tianero MD, Di Luccia B, Ahern PP, Merriman J, Cortez VS, Caparon MG, Donia MS, Gilfillan S, et al. (2017) Lactobacillus reuteri induces gut intraepithelial CD $4^{+} \mathrm{CD} 8 \alpha \alpha^{+}$T cells. Science 357:806-810.

Cheng J, Shah YM, and Gonzalez FJ (2012) Pregnane X receptor as a target for treatment of inflammatory bowel disorders. Trends Pharmacol Sci 33:323-330.
Chong J, Soufan O, Li C, Caraus I, Li S, Bourque G, Wishart DS, and Xia J (2018) MetaboAnalys 4.0: towards more transparent and integrative metabolomics analysis. Nucleic Acids Res 46 (W1):W486-W494.

Cole JT (2015) Metabolism of BCAAs, Branched Chain Amino Acids in Clinical Nutrition, Humana Press.

Cowell WJ, Sjödin A, Jones R, Wang Y, Wang S, and Herbstman JB (2019) Temporal trends and developmental patterns of plasma polybrominated diphenyl ether concentrations over a 15-year period between 1998 and 2013. J Expo Sci Environ Epidemiol 29:49-60.

Cowens KR, Simpson S, Thomas WK, and Carey GB (2015) Polybrominated diphenyl ether (PBDE)-induced suppression of phosphoenolpyruvate carboxykinase (PEPCK) decreases hepatic glyceroneogenesis and disrupts hepatic lipid homeostasis. J Toxicol Environ Health A 78: 1437-1449.

de Mello VD, Paananen J, Lindström J, Lankinen MA, Shi L, Kuusisto J, Pihlajamäki J, Auriola S, Lehtonen M, Rolandsson O, et al. (2017) Indolepropionic acid and novel lipid metabolites are associated with a lower risk of type 2 diabetes in the Finnish Diabetes Prevention Study. Sci Rep 7:46337.

Deng L, Gu H, Zhu J, Nagana Gowda GA, Djukovic D, Chiorean EG, and Raftery D (2016) Combining NMR and LC/MS using backward variable elimination: metabolomics analysis of colorectal cancer, polyps, and healthy controls. Anal Chem 88:7975-7983.

Dodd D, Spitzer MH, Van Treuren W, Merrill BD, Hryckowian AJ, Higginbottom SK, Le A Cowan TM, Nolan GP, Fischbach MA, et al. (2017) A gut bacterial pathway metabolizes aromatic amino acids into nine circulating metabolites. Nature 551:648-652.

Domingueti CP, Dusse LM, Carvalho M, de Sousa LP, Gomes KB, and Fernandes AP (2016) Diabetes mellitus: the linkage between oxidative stress, inflammation, hypercoagulability and vascular complications. J Diabetes Complications 30:738-745.

Donath MY and Shoelson SE (2011) Type 2 diabetes as an inflammatory disease. Nat Rev Immunol 11:98-107.

Dunnick JK, Brix A, Cunny H, Vallant M, and Shockley KR (2012) Characterization of polybrominated diphenyl ether toxicity in Wistar Han rats and use of liver microarray data for predicting disease susceptibilities. Toxicol Pathol 40:93-106.

Eslami B, Naddafi K, Rastkari N, Rashidi BH, Djazayeri A, and Malekafzali H (2016) Association between serum concentrations of persistent organic pollutants and gestational diabetes mellitus in primiparous women. Environ Res 151:706-712.

Farah R, Shurtz-Swirski R, and Lapin O (2008) Intensification of oxidative stress and inflammation in type 2 diabetes despite antihyperglycemic treatment. Cardiovasc Diabetol 7:20-20.

Girousse A, Tavernier G, Valle C, Moro C, Mejhert N, Dinel A-L, Houssier M, Roussel B, BessePatin A, Combes M, et al. (2013) Partial inhibition of adipose tissue lipolysis improves glucose metabolism and insulin sensitivity without alteration of fat mass. PLoS Biol 11:e1001485.

Goldfine AB, Fonseca V, and Shoelson SE (2011) Therapeutic approaches to target inflammation in type 2 diabetes. Clin Chem 57:162-167.

Gu H, Carroll PA, Du J, Zhu J, Neto FC, Eisenman RN, and Raftery D (2016) Quantitative method to investigate the balance between metabolism and proteome biomass: starting from glycine. Angew Chem Int Ed Engl 55:15646-15650.

Gu H, Zhang P, Zhu J, and Raftery D (2015) Globally optimized targeted mass spectrometry: reliable metabolomics analysis with broad coverage. Anal Chem 87:12355-12362.

Harrell F, Dupont C, , et al. [Internet] (2016) Hmisc. Available from: https://CRAN.R-project.org/ package $=$ Hmisc $($ Version 4.1-1 used $)$

Heimann E, Nyman M, and Degerman E (2014) Propionic acid and butyric acid inhibit lipolysis and de novo lipogenesis and increase insulin-stimulated glucose uptake in primary rat adipocytes. Adipocyte 4:81-88

Heimann E, Nyman M, Pålbrink A-K, Lindkvist-Petersson K, and Degerman E (2016) Branched short-chain fatty acids modulate glucose and lipid metabolism in primary adipocytes. Adipocyte 5 (4):359-368

Helaleh M, Diboun I, Al-Tamimi N, Al-Sulaiti H, Al-Emadi M, Madani A, Mazloum NA, Latiff A, and Elrayess MA (2018) Association of polybrominated diphenyl ethers in two fat compartments with increased risk of insulin resistance in obese individuals. Chemosphere 209:268-276.

Hites RA (2004) Polybrominated diphenyl ethers in the environment and in people: a meta-analysis of concentrations. Environ Sci Technol 38:945-956.

Hojs R, Ekart R, Bevc S, and Hojs N (2016) Markers of inflammation and oxidative stress in the development and progression of renal disease in diabetic patients. Nephron 133:159-162.

Hoppe AA and Carey GB (2007) Polybrominated diphenyl ethers as endocrine disruptors of adipocyte metabolism. Obesity (Silver Spring) 15:2942-2950.

Hothorn T, Bretz F, and Westfall P (2008) Simultaneous inference in general parametric models Biom J 50:346-363 Available from: https://CRAN.R-project.org/package=multcomp (Version $1.4-10$ used)

Hurley S, Goldberg D, Nelson DO, Guo W, Wang Y, Baek H-G, Park J-S, Petreas M, Bernstein L, Anton-Culver H, et al. (2017) Temporal evaluation of polybrominated diphenyl ether (PBDE) serum levels in middle-aged and older California women, 2011-2015. Environ Sci Technol 51:4697-4704.

Imm P, Knobeloch L, Buelow C, and Anderson HA (2009) Household exposures to polybrominated diphenyl ethers (PBDEs) in a Wisconsin Cohort. Environ Health Perspect 117: 1890-1895.

Kanehisa M, Furumichi M, Tanabe M, Sato Y, and Morishima K (2017) KEGG: new perspectives on genomes, pathways, diseases and drugs. Nucleic Acids Res 45 (D1):D353-D361.

Kanehisa M and Goto S (2000) KEGG: kyoto encyclopedia of genes and genomes. Nucleic Acids Res 28:27-30.

Kanehisa M, Sato Y, Kawashima M, Furumichi M, and Tanabe M (2016) KEGG as a reference resource for gene and protein annotation. Nucleic Acids Res 44 (D1):D457-D462.

Karandrea S, Yin H, Liang X, and Heart EA (2017) BDE-47 and BDE-85 stimulate insulin secretion in INS-1 832/13 pancreatic $\beta$-cells through the thyroid receptor and Akt. Environ Toxicol Pharmacol 56:29-34.

Koike E, Yanagisawa R, Takigami H, and Takano H (2014) Penta- and octa-bromodiphenyl ethers promote proinflammatory protein expression in human bronchial epithelial cells in vitro. Toxicol In Vitro 28:327-333.

Krumm EA, Patel VJ, Tillery TS, Yasrebi A, Shen J, Guo GL, Marco SM, Buckley BT, and Roepke TA (2018) Organophosphate flame-retardants alter adult mouse homeostasis and gene expression in a sex-dependent manner potentially through interactions with ER $\alpha$. Toxicol Sci 162:212-224

La Guardia MJ, Hale RC, and Harvey E (2006) Detailed polybrominated diphenyl ether (PBDE) congener composition of the widely used penta-, octa-, and deca-PBDE technical flame-retardant mixtures. Environ Sci Technol 40:6247-6254. 
Lenth RV (2016) Least-squares means: the R package lsmeans. J Stat Softw 69 Available from: https://CRAN.R-project.org/package= lsmeans (Version 2.27-62 used).

Li CY, Dempsey JL, Wang D, Lee S, Weigel KM, Fei Q, Bhatt DK, Prasad B, Raftery D, Gu H, et al. (2018) PBDEs altered gut microbiome and bile acid homeostasis in male C57BL/6 mice. Drug Metab Dispos 46:1226-1240.

Li CY, Lee S, Cade S, Kuo LJ, Schultz IR, Bhatt DK, Prasad B, Bammler TK, and Cui JY (2017) Novel interactions between gut microbiome and host drug-processing genes modify the hepatic metabolism of the environmental chemicals polybrominated diphenyl ethers. Drug Metab Dispos 45:1197-1214

Liebich HM and Först C (1984) Hydroxycarboxylic and oxocarboxylic acids in urine: product from branched-chain amino acid degradation and from ketogenesis. J Chromatogr 309 (2): 225-242, doi: 10.1016/0378-4347(84)80031-6 6434570.

Liu X, Zhan H, Zeng X, Zhang C, and Chen D (2012) The PBDE-209 exposure during pregnancy and lactation impairs immune function in rats. Mediators Inflamm 2012:692467.

Lu J-P, Knežević A, Wang Y-X, Rudan I, Campbell H, Zou Z-K, Lan J, Lai Q-X, Wu J-J, He Y, et al. (2011) Screening novel biomarkers for metabolic syndrome by profiling human plasma N-glycans in Chinese Han and Croatian populations. J Proteome Res 10 (11):4959-4969.

Lv Q-Y, Wan B, Guo L-H, Zhao L, and Yang Y (2015) In vitro immune toxicity of polybrominated diphenyl ethers on murine peritoneal macrophages: apoptosis and immune cell dysfunction. Chemosphere 120:621-630.

Lynch CJ and Adams SH (2014) Branched-chain amino acids in metabolic signalling and insulin resistance. Nat Rev Endocrinol 10 (12):723-736.

Ma W-L, Yun S, Bell EM, Druschel CM, Caggana M, Aldous KM, Buck Louis GM, and Kannan K (2013) Temporal trends of polybrominated diphenyl ethers (PBDEs) in the blood of newborns from New York State during 1997 through 2011: analysis of dried blood spots from the newborn screening program. Environ Sci Technol 47:8015-8021.

Marchitti SA, Fenton SE, Mendola P, Kenneke JF, and Hines EP (2017) Polybrominated diphenyl ethers in human milk and serum from the U.S. EPA MAMA Study: modeled predictions of infant exposure and considerations for risk assessment. Environ Health Perspect 125:706-713.

Martin PA, Mayne GJ, Bursian FS, Tomy G, Palace V, Pekarik C, and Smits J (2007) Immunotoxicity of the commercial polybrominated diphenyl ether mixture DE-71 in ranch mink (Mustela vison). Environ Toxicol Chem 26:988-997.

Mauvais-Jarvis F, Clegg DJ, and Hevener AL (2013) The role of estrogens in control of energy balance and glucose homeostasis. Endocr Rev 34:309-338.

McConnachie LA, Mohar I, Hudson FN, Ware CB, Ladiges WC, Fernandez C, ChattertonKirchmeier S, White CC, Pierce RH, and Kavanagh TJ (2007) Glutamate cysteine ligase modifier subunit deficiency and gender as determinants of acetaminophen-induced hepatotoxicity in mice. Toxicol Sci 99:628-636.

McIntyre RL, Kenerson HL, Subramanian S, Wang SA, Kazami M, Stapleton HM, and Yeung RS (2015) Polybrominated diphenyl ether congener, BDE-47, impairs insulin sensitivity in mice with liver-specific Pten deficiency. BMC Obes 2:3

McLachlan F, Timofeeva M, Bermingham M, Wild S, Rudan I, Lauc G, Wang W, Campbell H, Wilson J, and Theodoratou E (2016) A Case-control Study in an Orcadian Population Investigating the Relationship between Human Plasma N-glycans and Metabolic Syndrome. Journal of Glycomics \& Lipidomics 6 (3).

Morigny P, Houssier M, Mouisel E, and Langin D (2016) Adipocyte lipolysis and insulin resistance. Biochimie 125:259-266.

Nicholson JK, Holmes E, Kinross J, Burcelin R, Gibson G, Jia W, and Pettersson S (2012) Host-gut microbiota metabolic interactions. Science 336:1262-1267.

Pacyniak EK, Cheng X, Cunningham ML, Crofton K, Klaassen CD, and Guo GL (2007) The flame retardants, polybrominated diphenyl ethers, are pregnane X receptor activators. Toxicol Sci 97 $94-102$.

Parry E, Zota AR, Park J-S, and Woodruff TJ (2018) Polybrominated diphenyl ethers (PBDEs) and hydroxylated PBDE metabolites (OH-PBDEs): a six-year temporal trend in Northern California pregnant women. Chemosphere 195:777-783.

Quan EM, Kamiya Y, Kamiya D, Denic V, Weibezahn J, Kato K, and Weissman JS (2008) Defining the glycan destruction signal for endoplasmic reticulum-associated degradation. $\mathrm{Mo}$ Cell 32 (6):870-877.

R Core Team (2017) R: A Language and Environment for Statistical Computing, R Foundation for Statistical Computing, Vienna, Austria.

Reyes NL, Banks GB, Tsang M, Margineantu D, Gu H, Djukovic D, Chan J, Torres M, Liggitt HD, Hirenallur-S DK, et al. (2015) Fnip1 regulates skeletal muscle fiber type specification, fatigue resistance, and susceptibility to muscular dystrophy. Proc Natl Acad Sci USA 112:424-429.

Ross D and Siegel D (2018) NQO1 in protection against oxidative stress. Curr Opin Toxicol 7 $67-72$.

Sanders JM, Burka LT, Smith CS, Black W, James R, and Cunningham ML (2005) Differential expression of CYP1A, 2B, and 3A genes in the F344 rat following exposure to a polybrominated diphenyl ether mixture or individual components. Toxicol Sci 88:127-133.

Schecter A, Haffner D, Colacino J, Patel K, Päpke O, Opel M, and Birnbaum L (2010) Polybrominated diphenyl ethers (PBDEs) and hexabromocyclodecane (HBCD) in composite U.S. food samples. Environ Health Perspect 118:357-362.

Schecter A, Päpke O, Tung K-C, Staskal D, and Birnbaum L (2004) Polybrominated diphenyl ethers contamination of United States food. Environ Sci Technol 38:5306-5311.

Schecter A, Pavuk M, Päpke O, Ryan JJ, Birnbaum L, and Rosen R (2003) Polybrominated diphenyl ethers (PBDEs) in U.S. mothers' milk. Environ Health Perspect 111:1723-1729.

Scoville DK, Nolin JD, Ogden HL, An D, Afsharinejad Z, Johnson BW, Bammler TK, Gao X, Frevert CW, Altemeier WA, et al. (2019) Quantum dots and mouse strain influence house dust mite-induced allergic airway disease. Toxicol Appl Pharmacol 368:55-62.

Sjödin A, Patterson DG Jr, and Bergman A (2001) Brominated flame retardants in serum from U.S blood donors. Environ Sci Technol 35:3830-3833.

Smarr MM, Grantz KL, Zhang C, Sundaram R, Maisog JM, Barr DB, and Louis GMB (2016) Persistent organic pollutants and pregnancy complications. Sci Total Environ 551-552:285-291.
Søfteland L, Petersen K, Stavrum A-K, Wu T, and Olsvik PA (2011) Hepatic in vitro toxicity assessment of PBDE congeners BDE47, BDE153 and BDE154 in Atlantic salmon (Salmo salar L.). Aquat Toxicol 105:246-263.

Sood RF, Gu H, Djukovic D, Deng L, Ga M, Muffley LA, Raftery D, and Hocking AM (2015) Targeted metabolic profiling of wounds in diabetic and nondiabetic mice. Wound Repair Regen 23:423-434.

Sperber H, Mathieu J, Wang Y, Ferreccio A, Hesson J, Xu Z, Fischer KA, Devi A, Detraux D, Gu $\mathrm{H}$, et al. (2015) The metabolome regulates the epigenetic landscape during naive-to-primed human embryonic stem cell transition. Nat Cell Biol 17:1523-1535.

Sueyoshi T, Li L, Wang H, Moore R, Kodavanti PRS, Lehmler H-J, Negishi M, and Birnbaum LS (2014) Flame retardant BDE-47 effectively activates nuclear receptor CAR in human primary hepatocytes. Toxicol Sci 137:292-302.

Thiel C, Schwarz M, Hasilik M, Grieben U, Hanefeld F, Lehle L, von Figura K, and Körner C (2002) Deficiency of dolichyl-P-Man:Man7GlcNAc2-PP-dolichyl mannosyltransferase causes congenital disorder of glycosylation type Ig. Biochem J 367 (Pt 1):195-201.

Trapnell C, Williams BA, Pertea G, Mortazavi A, Kwan G, van Baren MJ, Salzberg SL, Wold BJ, and Pachter L (2010) Transcript assembly and quantification by RNA-Seq reveals unannotated transcripts and isoform switching during cell differentiation. Nat Biotechnol 28:511-515.

US-EPA (2012) Significant new use and test rules: certain polybrominated diphenylethers. Chemical Control Division, Office of Pollution Prevention and Toxics, Environmental Protection Agency, Washington, DC

Venkatesh M, Mukherjee S, Wang H, Li H, Sun K, Benechet AP, Qiu Z, Maher L, Redinbo MR, Phillips RS, et al. (2014) Symbiotic bacterial metabolites regulate gastrointestinal barrier function via the xenobiotic sensor PXR and Toll-like receptor 4. Immunity 41:296-310.

Vikram A, Tripathi DN, Kumar A, and Singh S (2014) Oxidative stress and inflammation in diabetic complications. Int J Endocrinol 2014:679754.

Warnes G, , Bolker B, , Bonebakker L, , Gentleman R, , Huber Andy Liaw W, Lumley T, Maechler M, Magnusson A, Moeller S, , Schwartz M, et al. (2013) gplots: Various R programming tools for plotting data. Available from: https://CRAN.R-project.org/ package $=$ gplots (Version 3.0.1.1 used).

Wei T and and Simko V [Internet] (2016) corrplot: visualization of a correlation matrix. Available from: https://CRAN.R-project.org/package $=$ corrplot (Version 0.84 used).

Weldy CS, White CC, Wilkerson H-W, Larson TV, Stewart JA, Gill SE, Parks WC, and Kavanagh TJ (2011) Heterozygosity in the glutathione synthesis gene Gclm increases sensitivity to diese exhaust particulate induced lung inflammation in mice. Inhal Toxicol 23:724-735.

White CC, Viernes H, Krejsa CM, Botta D, and Kavanagh TJ (2003) Fluorescence-based microtite plate assay for glutamate-cysteine ligase activity. Anal Biochem 318:175-180.

Wickham H (2009) ggplot2: Elegant Graphics for Data Analysis. Springer Publishing Company, New York, NY. Available from: https://CRAN.R-project.org/package=ggplot2 (Version 2.2.1 used)

Yisireyili M, Takeshita K, Saito S, Murohara T, and Niwa T (2017) Indole-3-propionic acid suppresses indoxyl sulfate-induced expression of fibrotic and inflammatory genes in proximal tubular cells. Nagoya J Med Sci 79:477-486.

Zarling EJ and Ruchim MA (1987) Protein origin of the volatile fatty acids isobutyrate and isovalerate in human stool. J Lab Clin Med 109 (5):566-570 3572204.

Zeng X, Massey Simonich SL, Robrock KR, KorytáR P, Alvarez-Cohen L, and Barofsky DF (2008) Development and validation of a congener-specific photodegradation model for polybrominated diphenyl ethers. Environ Toxicol Chem 27:2427-2435.

Zhang Z, Li S, Liu L, Wang L, Xiao X, Sun Z, Wang X, Wang C, Wang M, Li L, et al. (2016) Environmental exposure to BDE47 is associated with increased diabetes prevalence: evidence from community-based case-control studies and an animal experiment. Sci Rep 6:27854.

Zhang Z, Sun Z-Z, Xiao X, Zhou S, Wang X-C, Gu J, Qiu L-L, Zhang X-H, Xu Q, Zhen B, et al (2013) Mechanism of BDE209-induced impaired glucose homeostasis based on gene microarray analysis of adult rat liver. Arch Toxicol 87:1557-1567.

Zhou J, Chen DJ, Liao QP, and Yu YH (2006) [Impact of PBDE-209 exposure during pregnancy and lactation on immune function of offspring rats]. Nan Fang Yi Ke Da Xue Xue Bao 26: $738-741$.

Zhu J, Djukovic D, Deng L, Gu H, Himmati F, Abu Zaid M, Chiorean EG, and Raftery D (2015) Targeted serum metabolite profiling and sequential metabolite ratio analysis for colorectal cance progression monitoring. Anal Bioanal Chem 407:7857-7863.

Zhu J, Djukovic D, Deng L, Gu H, Himmati F, Chiorean EG, and Raftery D (2014) Colorectal cancer detection using targeted serum metabolic profiling. J Proteome Res 13:4120-4130.

Zota AR, Linderholm L, Park J-S, Petreas M, Guo T, Privalsky ML, Zoeller RT, and Woodruff TJ (2013) Temporal comparison of PBDEs, OH-PBDEs, PCBs, and OH-PCBs in the serum of second trimester pregnant women recruited from San Francisco General Hospital, California. Environ Sci Technol 47:11776-11784.

ATSDR [Internet] (2017) Toxicological profile for polybrominated diphenyl ethers (PBDEs) Agency for Toxic Substances and Disease Registry, Atlanta, GA. Available from: https://www. atsdr.cdc . gov/ToxProfiles/tp .asp?id=901\&tid=183.

EU [Internet] (2001) European Union Risk Assessment Report: Diphenyl Ether, Pentabromo Derivative(Pentabromodiphenyl Ether) Available from: https://echa.europa.eu/documents/10162/ 781ee1e9-6c90-467e-998b-8910ca2793e5.

Address correspondence to: Dr. Julia Yue Cui, Department of Environmental and Occupational Health Sciences, University of Washington, 4225 Roosevelt Way NE, Seattle, WA 98105. E-mail: juliacui@uw.edu; or Dr. Haiwei Gu, Arizona Metabolomics Laboratory, School of Nutrition and Health Promotion, College of Health Solutions, Arizona State University, Phoenix, AZ 85004. E-mail: haiweigu@ asu.edu 